\title{
Optimal Configuration of Tetrahedral Spacecraft Formations $^{1}$
}

\author{
Geoffrey T. Huntington, ${ }^{2}$ David Benson, ${ }^{3}$ and Anil V. Rao ${ }^{4}$
}

\begin{abstract}
The problem of determining minimum-fuel maneuver sequences for a four-spacecraft formation is considered. The objective of this paper is to find fuel-optimal spacecraft trajectories that transfer four spacecraft from an initial parking orbit to a desired terminal reference orbit while satisfying a set of constraints on the formation at the terminal time. Trajectories involving both one and two allowable maneuvers per spacecraft are considered. The resulting nonlinear optimal control problem is solved numerically using a recently developed direct transcription method called the Gauss pseudospectral method. The results presented in this paper highlight interesting features of the fuel-optimal formation and control. Furthermore, by showing that the discretized first-order optimality conditions from an indirect formulation are satisfied, a post-optimality analysis of the results demonstrates the accuracy and usefulness of the Gauss pseudospectral method.
\end{abstract}

\section{Introduction}

Spacecraft formation flying is defined as a set of more than one spacecraft whose states are coupled through a common control law [1], and has been identified as an enabling technology for many future space missions [2]. In particular, space missions using multiple spacecraft, as compared with using a single spacecraft, allow simultaneous measurements to be taken at specific relative locations, thereby improving science return. Critical to the successful implementation of formation flying missions is trajectory design (also called path planning or guidance). Formation flying trajectory design has two main categories: stationkeeping (i.e.,

${ }^{1}$ Presented as paper AAS 05-103 at the 2005 Space Flight Mechanics Conference in Copper Mountain, Colorado and paper AAS 05-339 at the 2005 Astrodynamics Specialist Conference in Lake Tahoe, California. ${ }^{2}$ Guidance \& Navigation Systems Engineer, The Jet Propulsion Laboratory, 4800 Oak Grove Drive, Pasadena, CA 91109. E-mail: Geoffrey.T.Huntington@jpl.nasa.gov.

${ }^{3}$ Senior member of the Technical Staff, The Charles Stark Draper Laboratory, 555 Technology Square, Cambridge, MA 02139. E-mail: dbenson@draper.com.

${ }^{4}$ Senior Member of the Technical Staff, Guidance, Navigation, and Control Systems Division, the Charles Stark Draper Laboratory. Currently, Assistant Professor, Department of Mechanical and Aerospace Engineering, University of Florida, P.O. Box 116250, Gainesville, FL, 32611-6250. E-mail: anilvrao@ufl.edu. 
maintaining a cluster of spacecraft in a specified formation for a certain portion of the trajectory) and reconfiguration (i.e., maneuvering a cluster of spacecraft from one formation to another formation).

In this paper we consider a specific reconfiguration problem, sometimes referred to as an orbit insertion problem or an initialization problem [3]. The objective is to determine the minimum-fuel trajectory and control that optimally transfers four spacecraft from an initial parking orbit to a terminal reference orbit while simultaneously satisfying a set of formation constraints at some point along the terminal reference orbit. In this research, the formation constraints place requirements on both the inter-spacecraft distance and the volume of the formation geometry. Given the desire to determine an optimal trajectory, the configuration problem is posed as an optimal control problem. Moreover, in this particular case the optimal control problem is highly nonlinear with no analytic solution and, thus, must be solved numerically.

This research focuses on the problem of determining optimal tetrahedral formations, a problem which has been considered previously. Much of the work in this area focuses on various ways to formulate and solve an optimal control problem that either initializes or reconfigures the tetrahedral formation. In reference [4], a hierarchical strategy is compared to a particle swarm approach to find optimal tetrahedral reconfiguration trajectories. In a similar application [5], a search space reduction technique is used to solve the formation optimal control problem. In these papers, significant simplifications are placed into the model or the search space in order to make the problem more manageable. In other approaches, the formation optimal control problem is parsed into segments $[3,6,7]$, where the segments are optimized individually and then patched together. While this technique makes the problem more tractable, it also reduces the solution search space and generally leads to suboptimal solutions. In this work the problem is formulated as a single unified numerical optimization procedure in order to encompass the entire search space.

Numerical methods for solving optimal control problems fall into two general categories: indirect methods and direct methods. An excellent survey of various numerical methods for solving optimal control problems can be found in reference [8]. In an indirect method, the optimal solution is found by solving a Hamiltonian boundary-value problem (HBVP) derived from the first-order necessary conditions for optimality [9]. The primary advantages of indirect methods are their high accuracy in the solution and the assurance that the solution satisfies the first-order optimality conditions. However, indirect methods have several disadvantages including possible difficulties in deriving the HBVP, small radii of convergence, and the requisite of a good initial guess for both the state and costate.

In a direct method, the continuous-time optimal control problem is transcribed to a nonlinear programming problem (NLP). The resulting NLP can be solved by welldeveloped algorithms and software. Direct methods have the advantage that the optimality conditions do not need to be derived [10]. Furthermore, they have much larger radii of convergence than indirect methods and thus, do not require as good of an initial guess. Lastly, for problems with path constraints, the switching structure of the constrained and unconstrained arcs does not need to be known a priori, unlike indirect methods. However, depending on the type of direct method, the solution may not contain any costate information, or may result in an inaccurate costate.

In recent years, considerable attention has been focused on a class of direct methods, called pseudospectral methods [11, 12, 13, 14, 15]. Pseudospectral methods 
in optimal control arose from spectral methods which were traditionally used to solve fluid dynamics problems $[11,15]$. In a pseudospectral method, the state and control are parameterized using a basis of global polynomials. The dynamics then are collocated at a specified set of points on the time interval of interest. While any set of unique collocation points can be chosen, in order to obtain the highest accuracy, the collocation points are chosen to be the roots of an orthogonal polynomial (or linear combinations of such polynomials and their derivatives). As a result, pseudospectral methods are generally implemented via orthogonal collocation (thus some researchers use the term pseudospectral $[12,16]$ while others use the term orthogonal collocation $[17,18])$. Seminal work in orthogonal collocation methods in optimal control date back to the work of reference [19] and some of the first work using orthogonal collocation methods in engineering can be found in the chemical engineering literature [17]. For smooth problems, pseudospectral methods typically have faster convergence rates than other methods, exhibiting so called "spectral accuracy" [20]. For nonsmooth problems or problems where modeling changes are desired, the optimal control problem can be divided into phases and orthogonal collocation can be applied globally within each phase. A vast amount of work has been done using pseudospectral methods to solve nonsmooth optimal control problems (see references [16, 17, 18, 21, 22, 23, 24]). Two previously developed and well known pseudospectral methods for solving optimal control problems are the Legendre pseudospectral method [12] (where Lagrange polynomials are used and collocation is performed at the Legendre-Gauss-Lobatto (LGL) points) and the Chebyshev pseudospectral method [25] (where Chebyshev polynomials are used and collocation is performed at the Chebyshev-Gauss-Lobatto (CGL) points). In addition, an enhancement to the Chebyshev pseudospectral method that uses a Clenshaw-Curtis quadrature scheme was developed in reference [14]. Finally, a costate estimation procedure that uses the Legendre pseudospectral method was developed in references [13] and [26, 27].

A recently developed pseudospectral method that has shown promise both in the solution and post-optimality analysis of optimally controlled systems is the Gauss pseudospectral method (GPM) [28, 29, 30]. In the Gauss pseudospectral method, the state is approximated using a basis of Lagrange polynomials and the optimal control problem is orthogonally collocated at the Legendre-Gauss $(L G)$ points. The Gauss pseudospectral method differs from other such methods in that the dynamics are not collocated at the boundary points. This choice of collocation, together with an appropriate Lagrange polynomial approximation of the costate, leads to KarushKuhn-Tucker (KKT) conditions that are identical to the discretized form of the first-order optimality conditions at the LG points. As a result, the solution of the NLP satisfies the same first-order conditions as an indirect solution that was obtained by solving the discretized HBVP conditions at the LG points. Furthermore, the equivalence between the KKT conditions and the discretized first-order necessary conditions leads to a method for estimating the costate of the continuoustime optimal control problem using the KKT multipliers of the NLP. Details of the Gauss pseudospectral method are found in reference [30].

In this research we are interested in implementing the Gauss pseudospectral method to solve a minimum-fuel four-spacecraft formation configuration problem $[31,32]$. Accurate numerical solutions are presented regarding the problem of configuring four spacecraft from an initial $600 \mathrm{~km}$ circular parking orbit to a terminal reference orbit of size $600 \mathrm{~km}$ by $7000 \mathrm{~km}$ altitude with the same inclination. More 
specifically, the four spacecraft must attain the apogee of the reference orbit while simultaneously placing the formation in an acceptable tetrahedron. Furthermore, the formation must also be repeatable each orbit which, in an inverse-square gravity field, is equivalent to requiring that the semimajor axis of each spacecraft be identical at the terminal time. Optimal trajectories are found for two scenarios: one where each spacecraft is limited to two maneuvers, and a second scenario where each spacecraft is limited to just one maneuver. For the purposes of this study it is assumed that the spacecraft are point masses in an inverse square gravity field. Furthermore, the dynamics are modeled using nonlinear modified equinoctial elements and the propulsion is modeled using finite thrust maneuvers.

This paper also provides an in-depth post-optimality analysis of the accuracy of the aforementioned optimal solution [33]. Specifically, the discrete control from the NLP is compared to the control computed from the first-order optimality conditions of the HBVP, discretized at the LG points. In order to make this comparison, a costate mapping principle is used [30]. Furthermore, a method for obtaining the control at the boundaries using the costate mapping is addressed. The results show that the control obtained from the Gauss pseudospectral method is in excellent agreement with the control predicted by the first-order optimality conditions of the discretized optimal control problem. This work highlights the accuracy and usefulness of the Gauss pseudospectral method as a way of solving nonlinear optimal control problems and verifying that the solution is indeed optimal.

\section{Overview of Spacecraft Formation Configuration Problem}

Consider the problem of maneuvering a fleet of four spacecraft from an initial parking orbit to a terminal reference orbit where the formation attains a desired tetrahedral shape at a specified point in the terminal reference orbit. For simplicity, assume that each spacecraft is initialized at the same point, (i.e., deployed simultaneously) on a circular orbit of altitude $h_{0}=600 \mathrm{~km}$ and inclination $i_{0}=28 \mathrm{deg}$. The desired terminal reference orbit is of size $600 \mathrm{~km}$ by $7000 \mathrm{~km}$ altitude, also with an inclination of $28 \mathrm{deg}$. The formation mesocenter, or mean position, must coincide with the apogee of this orbit at the final time. Although the spacecraft mesocenter does not follow Keplerian dynamics, the velocity of the mesocenter, or mean velocity, at the final time is constrained to correspond to the apogee orbit velocity to ensure the desired orbit is attained at the final time. Additional terminal constraints are placed on the spacecraft formation. The formation must be within 10 percent of a regular tetrahedron whose average spacecraft separation distance is between $4 \mathrm{~km}$ and $18 \mathrm{~km}$. Lastly, the semimajor axes of the four spacecraft must be equal to ensure periodic motion according to our dynamics model.

The trajectory is divided into five phases per spacecraft with the following phase sequence: coast, burn, coast, burn, and coast. Naturally, the dynamics are different for the coast and burn phases: there is no thrust during the coast phases, and in the burn phases the thrust is constant at its maximum value $T_{\max }$. Furthermore, at each phase interface the state must be continuous while the control can be discontinuous. Finally, the initial and terminal times of all phases are free (with the exception, of course, of the first phase where the initial time is fixed to zero). Figure 1 shows a schematic of the trajectory event sequence. This five-phase trajectory clearly has two maneuver opportunities. For the single-maneuver scenario, the fourth phase (or second burn phase) is constrained to zero duration. In the following three sections the configuration problem is explained in more detail. 


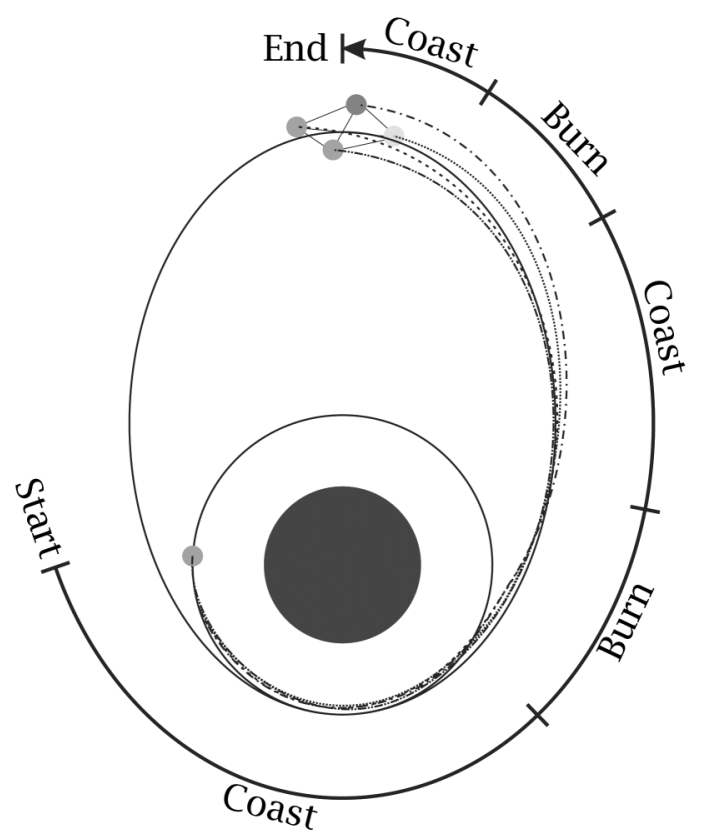

FIG. 1. Schematic of Trajectory Event Sequence.

\section{Spacecraft Model and Equations of Motion}

\section{Spacecraft Model}

In this application we consider four identical spacecraft each with a dry mass of $200 \mathrm{~kg}$ and a fuel mass of $300 \mathrm{~kg}$. Furthermore, the maximum thrust level of the engine is $7.015 \mathrm{kN}$ with an engine specific impulse of $285.7 \mathrm{~s}$. It is noted that these physical parameters are typical of a standard apogee kick motor [34]. Finally, all thrusting maneuvers are assumed to be non-impulsive.

\section{Dynamic Model During Thrust Phases}

During all thrust phases the state of each spacecraft is modeled using modified equinoctial elements. The three degree-of-freedom equations of motion for a spacecraft moving over a spherical nonrotating Earth are given in modified equinoctial elements as [35]

$$
\begin{aligned}
\dot{p}= & \frac{2 p}{w} \sqrt{\frac{p}{\mu}} a_{\theta} \\
\dot{P}_{1}= & \sqrt{\frac{p}{\mu}}\left\{-a_{r} \cos L+\left[(w+1) \sin L+P_{1}\right] \frac{a_{\theta}}{w}\right. \\
& \left.+\left[Q_{2} \sin L-Q_{1} \cos L\right] \frac{P_{2} a_{n}}{w}\right\} \\
\dot{P}_{2}= & \sqrt{\frac{p}{\mu}}\left\{a_{r} \sin L+\left[(w+1) \cos L+P_{2}\right] \frac{a_{\theta}}{w}\right. \\
& \left.-\left[Q_{2} \sin (L)-Q_{1} \cos L\right] \frac{P_{1} a_{n}}{w}\right\}
\end{aligned}
$$




$$
\begin{aligned}
\dot{Q}_{1} & =\sqrt{\frac{p}{\mu}}\left(\frac{s^{2}}{2 w}\right) a_{n} \sin L \\
\dot{Q}_{2} & =\sqrt{\frac{p}{\mu}}\left(\frac{s^{2}}{2 w}\right) a_{n} \cos L \\
\dot{L} & =\sqrt{\mu p}\left(\frac{w}{p}\right)^{2}+\sqrt{\frac{p}{\mu}} \frac{Q_{2} \sin L-Q_{1} \cos L}{w} a_{n}
\end{aligned}
$$

where $p$ is the semilatus rectum, $P_{1}, P_{2}, Q_{1}$, and $Q_{2}$ have no geometric definition, and $L$ is true longitude. Additionally, $w=1+P_{1} \sin L+P_{2} \cos L, s^{2}=1+Q_{1}^{2}+Q_{2}^{2}$, and $a_{r}, a_{\theta}$, and $a_{n}$ are the perturbing accelerations in the directions of $\mathbf{e}_{r}, \mathbf{e}_{\theta}$, and $\mathbf{e}_{n}$ where $\mathbf{e}_{r}$ is the unit vector in the radial direction, $\mathbf{e}_{n}$ is the unit vector in the direction normal to the orbital plane, and $\mathbf{e}_{\theta}=\mathbf{e}_{n} \times \mathbf{e}_{r}$ (thereby completing the righthanded system $\left.\left\{\mathbf{e}_{r}, \mathbf{e}_{\theta}, \mathbf{e}_{n}\right\}\right)$. For the application under consideration here, the perturbing accelerations are due entirely to thrust and can be written as

$$
a_{r}=\frac{T}{m} u_{r}, \quad a_{\theta}=\frac{T}{m} u_{\theta}, \quad a_{n}=\frac{T}{m} u_{n},
$$

where $T$ is the thrust magnitude, $m$ is the spacecraft mass, and $u_{r}, u_{\theta}$, and $u_{n}$ are the $\mathbf{e}_{r}, \mathbf{e}_{\theta}$, and $\mathbf{e}_{n}$ components, respectively, of the thrust direction. Finally, the mass flow rate of the engine is governed by the equation

$$
\dot{m}=-\frac{T}{g_{0} I_{s p}}
$$

where $g_{0}$ is the sea level acceleration due to gravity and $I_{s p}$ is the specific impulse of the engine.

\section{Dynamic Model for Coast Phases}

Since we are assuming a spherical Earth gravity model, during a coast phase the only equinoctial element that changes with time is the true longitude, $L$. In particular, during a coast phase the differential equation for the true longitude can be written as

$$
\dot{L}=\frac{\mathrm{d} L}{\mathrm{~d} t}=\frac{\sqrt{\mu p}}{p^{2}}\left(1+P_{1} \sin L+P_{2} \cos L\right)^{2}=f\left(L, p, P_{1}, P_{2}\right)
$$

Observing that all quantities except $L$ in equation (4) are constant, we can separate variables in equation (4) to give

$$
\mathrm{d} t=\frac{\mathrm{d} L}{f\left(L, p, P_{1}, P_{2}\right)}
$$

Integrating both sides of equation (5), we obtain

$$
t_{f}-t_{0}=\int_{L_{0}}^{L_{f}} \frac{\mathrm{d} L}{f\left(L, p, P_{1}, P_{2}\right)}
$$

where $t_{0}$ and $t_{f}$ are the initial and terminal time, respectively, of the coast phase while $L\left(t_{0}\right)=L_{0}$ and $L\left(t_{f}\right)=L_{f}$ are the initial and terminal true longitude. Since all other states are constant during a coast phase (again, because we have assumed 
spherical gravity), the dynamic model for each spacecraft during a coast phase is given as

$$
t_{f}-t_{0}-\int_{L_{0}}^{L_{f}} \frac{\mathrm{d} L}{f\left(L, p, P_{1}, P_{2}\right)}=0 \quad i=1, \ldots, 4
$$

All other components of the state (i.e., $p, P_{1}, P_{2}, Q_{1}, Q_{2}$, and $m$ ) are treated as constant optimization parameters. It is noted that, due to additional terminal constraints, the above model is not used for the final coast phase.

\section{Dynamic Model for Terminal Phase}

The terminal constraints in this problem focus largely on the relative position of the spacecraft, and are easily expressed in Cartesian Earth-centered inertial (ECI) coordinates. Therefore it makes sense to use a dynamic model that is consistent with the mathematical form of the constraints. Consequently, as opposed to modified equinoctial elements, the dynamics of each spacecraft in the final coast phase are described using ECI coordinates. This transition is by no means necessary, but it both reduces the complexity of the analytic derivatives of the equations of motion and exemplifies the versatility inherent in the Gauss pseudospectral method. The new dynamic model is given below as

$$
\dot{\mathbf{r}}=\mathbf{v} \quad \dot{\mathbf{v}}=-\mu \frac{\mathbf{r}}{\|\mathbf{r}\|^{3}}
$$

where $\mathbf{r}$ is the position vector measured from the center of the Earth, $\mathbf{v}$ is the inertial velocity, and $\mu$ is the gravitational parameter. In order to maintain continuity between the phases, the Cartesian variables are transformed to modified equinoctial elements at the start of the region of interest phase and are set equal to the elements at the final time of the previous phase via linkage conditions $[10,36]$ that are explained in the next section.

\section{Constraints}

\section{Initial Conditions}

All four spacecraft start in the same circular orbit at time $t=0$. The initial conditions corresponding to this orbit are given in orbital elements as

$$
\begin{array}{rlrl}
a(0) & =R_{e}+h_{0} & e(0) & =0 \\
i(0) & =28 \mathrm{deg} & \omega(0) & =270 \mathrm{deg} \\
\Omega(0) & =0 & v(0) & =270 \mathrm{deg}
\end{array}
$$

where $a$ is the semimajor axis, $e$ is the eccentricity, $i$ is the inclination, $\omega$ is the argument of perigee, $\Omega$ is the longitude of the ascending node, $v$ is the true anomaly, $R_{e}$ is the radius of the Earth, and $h_{0}=600 \mathrm{~km}$ is the initial altitude. It is noted that the initial argument of perigee, $\omega(0)$, is chosen to be the same as that of the terminal reference orbit while the initial true anomaly, $v(0)$, is arbitrary. The orbital elements in equation (9) are then converted to modified equinoctial elements using a transformation $T_{o 2 e}$ (see for example reference [35]) to obtain the initial state in modified equinoctial elements as

$$
\begin{aligned}
& p^{(i)}\left(t_{0}\right)=p_{0} \\
& P_{1}^{(i)}\left(t_{0}\right)=P_{1,0} \\
& P_{2}^{(i)}\left(t_{0}\right)=P_{2,0} \quad(i=1, \ldots, 4)
\end{aligned}
$$




$$
\begin{aligned}
Q_{1}^{(i)}\left(t_{0}\right) & =Q_{1,0} \\
Q_{2}^{(i)}\left(t_{0}\right) & =Q_{2,0} \\
L^{(i)}\left(t_{0}\right) & =L_{0}
\end{aligned}
$$

where $i$ is the $i$ th spacecraft. Furthermore, the initial mass of each spacecraft is equal to its maximum value, i.e.,

$$
m^{(i)}\left(t_{0}\right)=m_{\max } \quad(i=1, \ldots, 4)
$$

where $m_{\max }=500 \mathrm{~kg}$. Lastly it is noted that for this preliminary analysis, it is reasonable to assume that all four spacecraft can start at the same initial point without any conflict. However, if more realism is added to the problem, issues such as collision avoidance will have to be addressed. This will likely result in a slightly different initial condition and could affect the optimal trajectories as well.

\section{Interior Point Constraints}

In order for the state trajectory to be continuous at each phase interface, it is necessary to enforce linkage conditions at the phase boundaries. These linkage conditions are enforced on the modified equinoctial elements, mass, and time and are given as

$$
\begin{aligned}
& p^{(i)}\left(t_{f}^{(j)}\right)=p^{(i)}\left(t_{0}^{(j+1)}\right) \\
& P_{1}^{(i)}\left(t_{f}^{(j)}\right)=P_{1}^{(i)}\left(t_{0}^{(j+1)}\right) \\
& P_{2}^{(i)}\left(t_{f}^{(j)}\right)=P_{2}^{(i)}\left(t_{0}^{(j+1)}\right) \\
& Q_{1}^{(i)}\left(t_{f}^{(j)}\right)=Q_{1}^{(i)}\left(t_{0}^{(j+1)}\right) \quad(i=1, \ldots, 4) \\
& Q_{2}^{(i)}\left(t_{f}^{(j)}\right)=Q_{2}^{(i)}\left(t_{0}^{(j+1)}\right) \quad(j=1, \ldots, P-1) \\
& L^{(i)}\left(t_{f}^{(j)}\right)=L^{(i)}\left(t_{0}^{(j+1)}\right) \\
& m^{(i)}\left(t_{f}^{(j)}\right)=m^{(i)}\left(t_{0}^{(j+1)}\right) \\
& t_{f}^{(i)(j)}=t_{0}^{(i)(j+1)}
\end{aligned}
$$

where $j$ is the $j$ th phase and $P$ is the number of phases in the problem under consideration (in this case $P=5$ ). Finally, in order to ensure that time is increasing during the trajectory, an inequality constraint is placed on time during each phase as

$$
t_{f}^{(i)(j)}-t_{0}^{(i)(j)} \geq 0 \quad(i=1, \ldots, 4),(j=1, \ldots, P)
$$

In the case where each spacecraft has only one maneuver opportunity, equation (13) is an equality constraint for the fourth phase of each spacecraft.

\section{Path Constraints}

The following two path constraints are imposed on the four spacecraft. First, during the thrust phases of the trajectory it is necessary to constrain the thrust direction to be unit length. Defining the thrust direction as $\mathbf{u}_{T}=\left[\begin{array}{lll}u_{r} & u_{\theta} & u_{n}\end{array}\right]^{\mathrm{T}}$, a constraint is imposed on $\mathbf{u}_{T}$ during the thrust phases as

$$
\mathbf{u}_{T} \cdot \mathbf{u}_{T}=1
$$

Second, during flight the mass of each spacecraft cannot fall below the vehicle dry mass. Defining the dry mass of each vehicle as $m_{\text {dry }}$, an inequality constraint is imposed on the mass of each spacecraft during each phase of the trajectory

$$
m^{(i)} \geq m_{\mathrm{dry}}, \quad(i=1, \ldots, 4)
$$




\section{Terminal Constraints}

The position of the mesocenter of the formation is defined as

$$
\overline{\mathbf{r}}=\frac{1}{4} \sum_{i=1}^{4} \mathbf{r}^{(i)}
$$

where $\mathbf{r}^{(i)}$ is the position of the $i$ th spacecraft as measured from the center of the Earth. The first constraint imposed on the formation at $t=t_{f}$ is that the mesocenter must coincide with the apogee of the reference orbit. Defining the position of the reference orbit apogee as $\mathbf{r}_{a}$, the constraint imposed on the position of the formation mesocenter at $t=t_{f}$ is given as

$$
\overline{\mathbf{r}}\left(t_{f}\right)=\mathbf{r}_{a}
$$

Similarly, the velocity of the mesocenter of the formation is defined as

$$
\overline{\mathbf{v}}=\frac{1}{4} \sum_{i=1}^{4} \mathbf{v}^{(i)}
$$

where $\mathbf{v}^{(i)}$ is the velocity of the $i$ th spacecraft. Since six orbital elements are necessary to completely define an orbit's characteristics, constraining both the position and velocity of the mesocenter at $t=t_{f}$ ensures that the mesocenter is on the reference orbit for that instant in time. Defining the velocity of the reference orbit apogee as $\mathbf{v}_{a}$, the constraint imposed on the velocity of the formation mesocenter is given as

$$
\overline{\mathbf{v}}\left(t_{f}\right)=\mathbf{v}_{a}
$$

Next, the formation must form a tetrahedron upon reaching apogee of the final orbit. To ensure the quality of this tetrahedron, a set of constraints are formed that take into account both the formation size and shape. First, it is noted that the spacecraft must be within a certain distance of each other to take useful measurements. Although an average inter-spacecraft separation of $10 \mathrm{~km}$ is considered ideal, acceptable science return is still possible for average separations ranging from $4 \mathrm{~km}$ to $18 \mathrm{~km}$ [37]. For this application, the formation size is constrained by placing bounds on the formation's average side length, $\bar{L}$.

$$
4 \leq \bar{L} \leq 18
$$

In addition to the formation size, the formation shape must meet certain performance criteria. The metric used to determine the quality of the shape of the formation is called the $Q_{R 8}$ Geometric Factor [38], and is given as

$$
Q_{R 8}=V_{a} / V^{*}
$$

where $V_{a}$ is the actual volume of the tetrahedron, and $V^{*}$ is the (ideal) volume of a regular tetrahedron whose side length is $\bar{L}$. It is seen that the $Q_{R 8}$ metric contains no sensitivity to the size of the tetrahedron, hence the need for a separate sizing constraint. For this work, values of $Q_{R 8}$ greater than 0.9 are considered acceptable. Consequently, a terminal constraint is enforced as

$$
Q_{R 8} \geq 0.9
$$

See reference [38] for details on how to compute the $Q_{R 8}$ Geometric Factor. Next, in the absence of perturbations, a spacecraft's orbit period is solely a function of semimajor axis. In this case, spacecraft with equal semimajor axes (and therefore 
equal periods) will return to their relative positions exactly one period later. Consequently, the formation will be periodic if all spacecraft involved have the same semimajor axis. If perturbations are introduced to the model, there will be some degradation in the tetrahedron shape over time, but in general the degradation rate is reduced if the orbit periods are initially equal. Therefore if repeatability is desirable (as is usually the case), it is beneficial to constrain the semimajor axis of each spacecraft to be equal at the reference apogee, namely

$$
a^{(1)}\left(t_{f}^{(P)}\right)=a^{(2)}\left(t_{f}^{(P)}\right)=a^{(3)}\left(t_{f}^{(P)}\right)=a^{(4)}\left(t_{f}^{(P)}\right)
$$

Finally, it is required that the trajectories of all four spacecraft terminate at the same time, i.e.

$$
t_{f}^{(1)(P)}=t_{f}^{(2)(P)}=t_{f}^{(3)(P)}=t_{f}^{(4)(P)}
$$

where $t_{f}$ is free.

\section{Spacecraft Configuration Optimal Control Problem}

The spacecraft configuration optimal control problem is now stated formally. Using the aforementioned trajectory event sequence, determine the thrust profile that maximizes the sum of the terminal masses of each spacecraft, i.e., maximize the objective functional

$$
J=\sum_{i=1}^{4} m^{(i)}\left(t_{f}^{(P)}\right)
$$

subject to the differential equation constraints of equation (1) and equation (3), the initial constraints of equation (10) and equation (11), the interior point constraints of equation (12) and equation (13), the path constraints of equations (14) and (15), and the terminal constraints of equation (17), equation (19), equation (20), and equations (22)-(24).

\section{Continuous Bolza Problem}

The aforementioned optimal control problem can be written in the following general form [39, 40]. Determine the state, $\mathbf{x}(\tau) \in \mathbb{R}^{n}$, control, $\mathbf{u}(\tau) \in \mathbb{R}^{m}$, initial time, $t_{0}$, and final time, $t_{f}$, that minimize the cost functional

$$
J=\Phi\left(\mathbf{x}(-1), t_{0}, \mathbf{x}(1), t_{f}\right)+\frac{t_{f}-t_{0}}{2} \int_{-1}^{1} g\left(\mathbf{x}(\tau), \mathbf{u}(\tau), \tau ; t_{0}, t_{f}\right) \mathrm{d} \tau
$$

subject to the constraints

$$
\begin{aligned}
& \frac{\mathrm{d} \mathbf{x}}{\mathrm{d} \tau}=\frac{t_{f}-t_{0}}{2} \mathbf{f}\left(\mathbf{x}(\tau), \mathbf{u}(\tau), \tau ; t_{0}, t_{f}\right) \\
& \boldsymbol{\phi}\left(\mathbf{x}(-1), t_{0}, \mathbf{x}(1), t_{f}\right)=\mathbf{0} \in \mathbb{R}^{q} \\
& \mathbf{C}\left(\mathbf{x}(\tau), \mathbf{u}(\tau), \tau ; t_{0}, t_{f}\right) \leq \mathbf{0} \in \mathbb{R}^{c}
\end{aligned}
$$

The optimal control problem of equations (26)-(29) will be referred to as the continuous Bolza problem. It is noted that the optimal control problem of 
equations (26)-(29) is stated in terms of $\tau \in[-1,1]$ in order to apply the Gauss pseudospectral method. This formulation can be transformed from the time interval $\tau \in[-1,1]$ to the time interval $t \in\left[t_{0}, t_{f}\right]$ via the affine transformation

$$
t=\frac{t_{f}-t_{0}}{2} \tau+\frac{t_{f}+t_{0}}{2}
$$

\section{First-Order Necessary Conditions of Continuous Bolza Problem}

The indirect approach to solving the continuous Bolza problem of equations (26)-(29) in the previous section is to apply the calculus of variations and Pontryagin's Maximum Principle [41] to obtain first-order necessary conditions for optimality [40]. These variational conditions are typically derived using the augmented Hamiltonian, $\mathcal{H}$, defined as

$$
\begin{aligned}
\mathcal{H}\left(\mathbf{x}, \boldsymbol{\lambda}, \boldsymbol{\mu}, \mathbf{u}, \tau ; t_{0}, t_{f}\right)= & g\left(\mathbf{x}, \mathbf{u}, \tau ; t_{0}, t_{f}\right)+\boldsymbol{\lambda}^{\mathrm{T}}(\tau) \mathbf{f}\left(\mathbf{x}, \mathbf{u}, \tau ; t_{0}, t_{f}\right) \\
& -\boldsymbol{\mu}^{\mathrm{T}}(\tau) \mathbf{C}\left(\mathbf{x}, \mathbf{u}, \tau ; t_{0}, t_{f}\right)
\end{aligned}
$$

where $\boldsymbol{\lambda}(\tau) \in \mathbb{R}^{n}$ is the costate and $\boldsymbol{\mu}(\tau) \in \mathbb{R}^{c}$ is the Lagrange multiplier associated with the path constraint. The continuous-time HBVP conditions can be shown to be

$$
\begin{aligned}
\frac{d \mathbf{x}^{\mathrm{T}}}{d \tau} & =\frac{t_{f}-t_{0}}{2} \mathbf{f}\left(\mathbf{x}, \mathbf{u}, \tau ; t_{0}, t_{f}\right)=\frac{t_{f}-t_{0}}{2} \frac{\partial \mathcal{H}}{\partial \boldsymbol{\lambda}} \\
\frac{d \boldsymbol{\lambda}^{\mathrm{T}}}{d \tau} & =\frac{t_{f}-t_{0}}{2}\left(-\frac{\partial g}{\partial \mathbf{x}}-\boldsymbol{\lambda}^{\mathrm{T}} \frac{\partial \mathbf{f}}{\partial \mathbf{x}}+\boldsymbol{\mu}^{\mathrm{T}} \frac{\partial \mathbf{C}}{\partial \mathbf{x}}\right)=-\frac{t_{f}-t_{0}}{2} \frac{\partial \mathcal{H}}{\partial \mathbf{x}} \\
\mathbf{0}^{\mathrm{T}} & =\frac{\partial g}{\partial \mathbf{u}}+\boldsymbol{\lambda}^{\mathrm{T}} \frac{\partial \mathbf{f}}{\partial \mathbf{u}}-\boldsymbol{\mu}^{\mathrm{T}} \frac{\partial C}{\partial \mathbf{u}}=\frac{\partial \mathcal{H}}{\partial \mathbf{u}} \\
\boldsymbol{\phi}\left(\mathbf{x}\left(\tau_{0}\right), t_{0}, \mathbf{x}\left(\tau_{f}\right), t_{f}\right)=\mathbf{0} & \\
\boldsymbol{\lambda}^{\mathrm{T}}\left(\tau_{0}\right) & =-\frac{\partial \Phi}{\partial \mathbf{x}\left(\tau_{0}\right)}+\boldsymbol{v}^{\mathrm{T}} \frac{\partial \boldsymbol{\phi}}{\partial \mathbf{x}\left(\tau_{0}\right)}, \boldsymbol{\lambda}^{\mathrm{T}}\left(\tau_{f}\right)=\frac{\partial \Phi}{\partial \mathbf{x}\left(\tau_{f}\right)}-\boldsymbol{v}^{\mathrm{T}} \frac{\partial \boldsymbol{\phi}}{\partial \mathbf{x}\left(\tau_{f}\right)} \\
\mathcal{H}\left(t_{0}\right) & =\frac{\partial \Phi}{\partial t_{0}}-\boldsymbol{v}^{\mathrm{T}} \frac{\partial \boldsymbol{\phi}}{\partial t_{0}}, \mathcal{H}\left(t_{f}\right)=-\frac{\partial \Phi}{\partial t_{f}}+\boldsymbol{v}^{\mathrm{T}} \frac{\partial \boldsymbol{\phi}}{\partial t_{f}} \\
\mu_{j}(\tau) & =0, \text { when } \quad C_{j}\left(\mathbf{x}, \mathbf{u}, \tau ; t_{0}, t_{f}\right)<0, \quad j=1, \ldots, c \\
\mu_{j}(\tau) & \leq 0, \text { when } \quad C_{j}\left(\mathbf{x}, \mathbf{u}, \tau ; t_{0}, t_{f}\right)=0, \quad j=1, \ldots, c
\end{aligned}
$$

where $v \in \mathbb{R}^{q}$ is the Lagrange multiplier associated with the boundary condition $\boldsymbol{\phi}$. If one were to solve an optimal control problem via an indirect method, these are the first-order necessary equations for an optimal solution. The Gauss pseudospectral method does not evaluate these conditions explicitly, but it is shown in reference [30] that the conditions, when discretized via the Gauss pseudospectral method, are satisfied at the optimal solution.

\section{Gauss Pseudospectral Discretization of Continuous Bolza Problem}

The direct approach to solving the continuous Bolza optimal control problem is to discretize and transcribe equations (26)-(29) to a nonlinear programming 
problem (NLP) [30]. The Gauss pseudospectral method, like Legendre and Chebyshev methods, is based on approximating the state and control using interpolating polynomials. The state is approximated across each phase by using a basis of $N+1$ Lagrange interpolating polynomials [42], $\mathcal{L}_{i}(\tau)(i=0, \ldots, N)$,

$$
\mathbf{x}(\tau) \approx \mathbf{X}(\tau)=\sum_{i=0}^{N} \mathbf{X}\left(\tau_{i}\right) \mathcal{L}_{i}(\tau), \quad \text { where } \quad \mathcal{L}_{i}(\tau)=\prod_{j=0, j \neq i}^{N} \frac{\tau-\tau_{j}}{\tau_{i}-\tau_{j}}
$$

Additionally, the control is approximated across each phase using a basis of $N$ Lagrange interpolating polynomials $\mathcal{L}_{i}^{*}(\tau),(i=1, \ldots, N)$ as

$$
\mathbf{u}(\tau) \approx \mathbf{U}(\tau)=\sum_{i=1}^{N} \mathbf{U}\left(\tau_{i}\right) \mathcal{L}_{i}^{*}(\tau), \quad \text { where } \quad \mathcal{L}_{i}^{*}(\tau)=\prod_{j=1, j \neq i}^{N} \frac{\tau-\tau_{j}}{\tau_{i}-\tau_{j}}
$$

The derivative of each Lagrange polynomial corresponding to the state at the LG points can be represented in a differential approximation matrix, $D \in \mathbb{R}^{N \times N+1}$. The elements of the differential approximation matrix are determined offline as

$$
D_{k i}=\dot{\mathcal{L}}_{i}\left(\tau_{k}\right)=\sum_{l=0}^{N} \frac{\prod_{j=0, j \neq i, l}^{N}\left(\tau_{k}-\tau_{j}\right)}{\prod_{j=0, j \neq i}^{N}\left(\tau_{i}-\tau_{j}\right)}
$$

where $k=1, \ldots, N$ and $i=0, \ldots, N$. The dynamic state constraints are transcribed into algebraic constraints via the differential approximation matrix as

$$
\sum_{i=0}^{N} D_{k i} \mathbf{X}_{i}-\frac{t_{f}-t_{0}}{2} \mathbf{f}\left(\mathbf{X}_{k}, \mathbf{U}_{k} \tau_{k} ; t_{0}, t_{f}\right)=\mathbf{0} \quad(k=1, \ldots, N)
$$

where $\mathbf{X}_{k} \equiv \mathbf{X}\left(\tau_{k}\right) \in \mathbb{R}^{n}$ and $\mathbf{U}_{k} \equiv \mathbf{U}\left(\tau_{k}\right) \in \mathbb{R}^{m}(k=1, \ldots, N)$. Note that the dynamic constraint is collocated only at the LG points and not at the boundary points (this form of collocation differs from other well known pseudospectral methods such as those found in references [12] and [25]). Additional variables in the discretization are $\mathbf{X}_{0} \equiv \mathbf{X}(-1)$ and $\mathbf{X}_{f}$, where $\mathbf{X}_{f}$ is defined in terms of $\mathbf{X}_{k}$, $(k=0, \ldots, N)$ and $\mathbf{U}\left(\tau_{k}\right)(k=1, \ldots, N)$ via the Gauss quadrature [43]

$$
\mathbf{X}_{f} \equiv \mathbf{X}_{0}+\frac{t_{f}-t_{0}}{2} \sum_{k=1}^{N} w_{k} \mathbf{f}\left(\mathbf{X}_{k}, \mathbf{U}_{k}, \tau_{k} ; t_{0}, t_{f}\right)
$$

where $w_{k}$ are the Gauss weights. The continuous cost function of equation (26) is approximated using a Gauss quadrature as

$$
J=\Phi\left(\mathbf{X}_{0}, t_{0}, \mathbf{X}_{f}, t_{f}\right)+\frac{t_{f}-t_{0}}{2} \sum_{k=1}^{N} w_{k} g\left(\mathbf{X}_{k}, \mathbf{U}_{k}, \tau_{k} ; t_{0}, t_{f}\right)
$$

Next, the boundary constraint of equation (28) is expressed as

$$
\boldsymbol{\phi}\left(\mathbf{X}_{0}, t_{0}, \mathbf{X}_{f}, t_{f}\right)=\mathbf{0}
$$

Furthermore, the path constraint of equation (29) is evaluated at the LG points as

$$
\mathbf{C}\left(\mathbf{X}_{k}, \mathbf{U}_{k}, \tau_{k} ; t_{0}, t_{f}\right) \leq \mathbf{0} \quad(k=1, \ldots, N)
$$


The cost function of equation (38) and the algebraic constraints of equations (36), (37), (39), and (40) define a NLP whose solution is an approximate solution to the continuous Bolza problem. Finally, it is noted that discontinuities in the state or control can be handled efficiently by dividing the trajectory into phases, where the dynamics are transcribed within each phase and then connected together by additional phase interface (a.k.a linkage) constraints [23, 24].

\section{Costate Estimate}

Recall that the NLP is an approximation to the original optimal control problem, thus a solution satisfying the Karush-Kuhn-Tucker (KKT) conditions of the NLP in the previous section is not necessarily a solution to the original optimal control problem. However, confidence can be gained in a solution if it also satisfies the discretized first-order necessary conditions of the respective HBVP. As explained in reference [30], when using the Gauss pseudospectral method, there exists a mapping that relates exactly the KKT conditions of the NLP to the necessary conditions of the HBVP. This mapping, in terms of the KKT multipliers, $\tilde{\boldsymbol{\Lambda}}_{k}, \tilde{\boldsymbol{\mu}}_{k}, \tilde{\boldsymbol{\Lambda}}_{0}, \tilde{\boldsymbol{\Lambda}}_{F}$, and $\tilde{\boldsymbol{v}}$, is described as

$$
\begin{gathered}
\boldsymbol{\Lambda}_{k}=\frac{\tilde{\boldsymbol{\Lambda}}_{k}}{w_{k}}+\tilde{\boldsymbol{\Lambda}}_{F} \\
\boldsymbol{\mu}_{k}=\frac{2}{t_{f}-t_{0}} \frac{\tilde{\boldsymbol{\mu}}_{k}}{w_{k}} \\
\boldsymbol{\Lambda}_{0}=\tilde{\boldsymbol{v}} \tilde{\boldsymbol{\Lambda}}_{0}, \quad \boldsymbol{\Lambda}_{f}=\tilde{\boldsymbol{\Lambda}}_{F},
\end{gathered}
$$

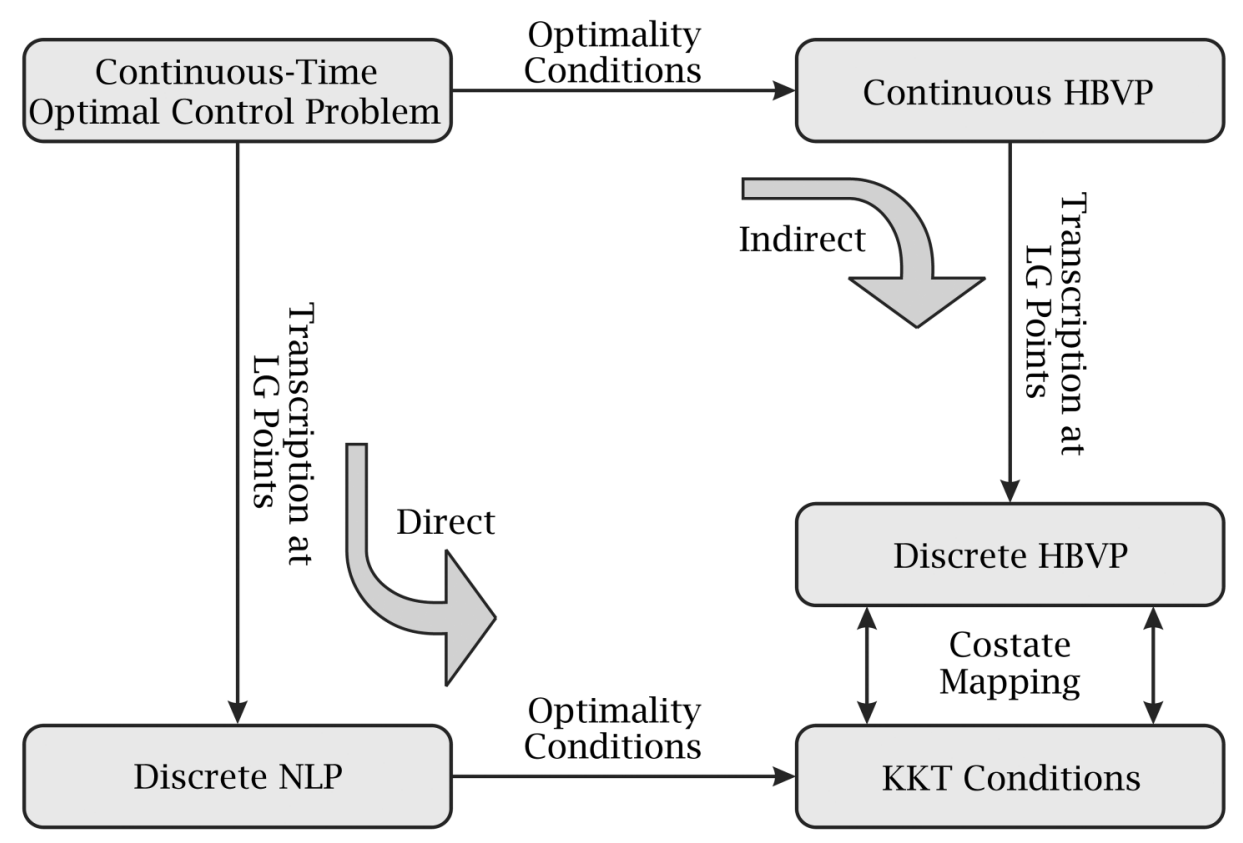

FIG. 2. Gauss Pseudospectral Discretization. 
The variables $\boldsymbol{\Lambda}_{0}, \boldsymbol{\Lambda}_{k}, \boldsymbol{\Lambda}_{f}, \boldsymbol{\mu}_{k}$, and $\boldsymbol{v}$, now defined in terms of NLP multipliers, can be substituted into the HBVP equations to ensure the optimality conditions have been met (this is demonstrated later in the paper). To illustrate this crucial concept, Fig. 2 shows the solution path for both the direct and indirect formulations. The discrete costate is then used to construct a continuous-time approximation to the costate, which is represented by a slightly different basis of $N+1$ Lagrange interpolating polynomials, defined as

$$
\boldsymbol{\lambda}(\tau) \approx \boldsymbol{\Lambda}(\tau)=\sum_{i=1}^{N} \boldsymbol{\Lambda}\left(\tau_{i}\right) \mathcal{L}_{i}^{\dagger}(\tau)+\boldsymbol{\Lambda}(1) \mathcal{L}_{N+1}^{\dagger}(\tau)
$$

where

$$
\mathcal{L}_{i}^{\dagger}(\tau)=\prod_{j=1, j \neq i}^{N+1} \frac{\tau-\tau_{j}}{\tau_{i}-\tau_{j}}, \quad(i=1, \ldots, N+1)
$$

Recall that the state is approximated using $N+1$ Lagrange polynomials based on $N$ LG points and the initial time, while the costate, $\boldsymbol{\lambda}$, is approximated using $N+1$ Lagrange polynomials consisting of the $N$ LG points and the final time. This discrepancy is required to preserve the mapping, and the reader can find more detailed information in reference [30]. The next section shows how this mapping can be used to determine an accurate control at the boundaries.

\section{Computation of Boundary Control}

Recall that the solution to the NLP described earlier contains a value for the control at each LG point, but not at the boundaries. These values are then inserted into the pseudospectral approximation for the control in equation (34) to create a continuous control across the entire interval. The initial and final control can be extrapolated from this control, but these values tend to be less accurate than the control at the LG points where the constraints were strictly enforced in the NLP. This section will show that by using the equivalence between the KKT conditions and HBVP optimality conditions, a better approximation to the boundary control can be determined.

First, one might ask, if the control is approximated using equation (34), how can one change this approximation in post-processing without disrupting the original NLP? The response to this question requires us to take a step back and see that equation (34) is not the only equation that will maintain the proper GPM transcription to an NLP. In the NLP, the control is discrete, and requires no derivative information, unlike the state. Any approximation that passes through the LG points is a legitimate approximation. Historically, equation (34) has been used to represent the control, but this is by no means required. For example, if a piecewise linear approximation to the control is used, it would result in an identical NLP. Therefore, if a continuous control can be found that equals the NLP solution at the LG points and also utilizes more information to get a better approximation at non-LG points, then this approximation can and should be used.

The equivalence of the HBVP and KKT conditions allows an alternative way to obtain information about the control at the boundaries. Recall that accurate state and costate information is available at the boundaries. Therefore an accurate approximation for the boundary control can be computed using the HBVP optimality 
conditions. These equations utilize the initial and final costate estimates, $\boldsymbol{\Lambda}_{0}$ and $\boldsymbol{\Lambda}_{f}$, obtained from the costate mapping in the previous section.

To obtain the estimate for the initial control, the HBVP equations involving $\mathbf{U}_{0}$ must be pinpointed and used to solve for the boundary control. In the case of the current formation flying problem, the HBVP equations involving $\mathbf{U}_{0}$ are determined from the partial derivative of the augmented Hamiltonian with respect to the control at $\tau_{0}=-1$ (i.e., $[\partial \mathcal{H} / \partial \mathbf{u}]_{\tau=\tau_{0}}$ ) and the complementary slackness condition. Notice that both the initial control, $\mathbf{U}_{0}$, and the multiplier associated with the path constraint, $\boldsymbol{\mu}_{0}$, are unknown (since the path constraint was only evaluated at the interior points in the NLP). These two quantities can be found by solving the system of $c+m$ equations for $\boldsymbol{\mu}_{0} \in \mathbb{R}^{c}$ and $\mathbf{U}_{0} \in \mathbb{R}^{m}$

$$
\begin{aligned}
{\left[\frac{\partial g}{\partial \mathbf{U}}\right]_{\tau=\tau_{0}}+\boldsymbol{\Lambda}_{0}^{\mathrm{T}}\left[\frac{\partial \mathbf{f}}{\partial \mathbf{U}}\right]_{\tau=\tau_{0}}-\boldsymbol{\mu}_{0}^{\mathrm{T}}\left[\frac{\partial \mathbf{C}}{\partial \mathbf{U}}\right]_{\tau=\tau_{0}} } & =\mathbf{0}^{\mathrm{T}} \\
\boldsymbol{\mu}_{0}^{\mathrm{T}} \mathbf{C}\left(\mathbf{U}_{0}, \tau_{0}\right) & =0
\end{aligned}
$$

Similarly, the control and multiplier at the terminal time $\tau_{f}=1$ can be found by solving the system of $c+m$ equations for $\boldsymbol{\mu}_{f} \in \mathbb{R}^{c}$ and $\mathbf{U}_{f} \in \mathbb{R}^{m}$

$$
\begin{aligned}
{\left[\frac{\partial g}{\partial \mathbf{U}}\right]_{\tau=\tau_{f}}+\boldsymbol{\Lambda}_{f}^{\mathrm{T}}\left[\frac{\partial \mathbf{f}}{\partial \mathbf{U}}\right]_{\tau=\tau_{f}}-\boldsymbol{\mu}_{f}^{\mathrm{T}}\left[\frac{\partial \mathbf{C}}{\partial \mathbf{U}}\right]_{\tau=\tau_{f}} } & =\mathbf{0}^{\mathrm{T}} \\
\boldsymbol{\mu}_{f}^{\mathrm{T}} \mathbf{C}\left(\mathbf{U}_{f}, \tau_{f}\right) & =0
\end{aligned}
$$

It is noted that empirical evidence has shown that the boundary control obtained via the HBVP equations is more accurate than the boundary control extrapolated from the pseudospectral approximation. This is largely due to the high accuracy in the initial and final costate [30].

\section{Numerical Solution via Gauss Pseudospectral Method}

The spacecraft configuration problem as described in the first sections of this paper is solved using the aforementioned Gauss pseudospectral method of reference [30]. The optimization was carried out with the MATLAB mex interface of the NLP solver SNOPT [44] using analytic first-order derivatives for both the constraint Jacobian and the gradient of the objective function. Furthermore, the optimal control problem was scaled canonically from SI units to a set of units such that the length scale is the radius of the Earth, the time scale is one Schuler period, and the mass scale is equal to the initial spacecraft mass. For this problem, 15 nodes (i.e., $13 \mathrm{LG}$ points) were used to approximate the trajectory in the burn phases. Since the first two coast phases utilize either static variables or a simple quadrature constraint, a pseudospectral approximation is not necessary for those phases. The quadrature can be computed numerically from the initial and final state; therefore only two nodes were needed in these coast phases. The final coast phase, which is calculated in ECI coordinates instead of equinoctial elements, requires a pseudospectral approximation. Because the duration of the final coast phase is unknown a priori, 40 nodes (i.e., 38 LG points) were used in the final coast phase. Note that these node amounts were chosen in order to accurately approximate the solution yet still maintain a reasonably sized NLP. In the future it is likely that the number of nodes per phase could become an optimization variable itself. In fact, some more 
mature optimization software codes such as SOCS [10] do have this feature, but this ability has not been developed for the GPM.

\section{Results}

The key results for both the single-maneuver and two-maneuver versions of the configuration problem are discussed in this section. The first subsection summarizes the results for the two-maneuver configuration problem, and the second subsection summarizes the results for the single-maneuver configuration problem. Lastly, the third subsection provides a detailed post-optimality analysis of the single-maneuver problem solution.

\section{Two-Maneuver Solution}

Recall that the formation must attain a geometry that is within $10 \%$ of a regular tetrahedron (measured by the quality factor) and have an average inter-spacecraft distance between 4 and $18 \mathrm{~km}$ at the terminal time. Furthermore, the spacecraft mesocenter must have a position and velocity that corresponds to the apogee of the reference orbit, and the spacecraft must have equal semimajor axes and terminal times. The solution from the NLP does indeed satisfy all these constraints, and interestingly, the minimum-fuel formation resides at the lower bound for both the volume constraint and average inter-spacecraft distance. This result is largely intuitive, since maneuvering the spacecraft to a larger separation distance would naturally require more fuel, and is counter-productive to the given cost function. Figure 3 provides a three-dimensional perspective of the terminal tetrahedron where $x, y$, and $z$ represent the standard Earth-centered inertial coordinate system [45].

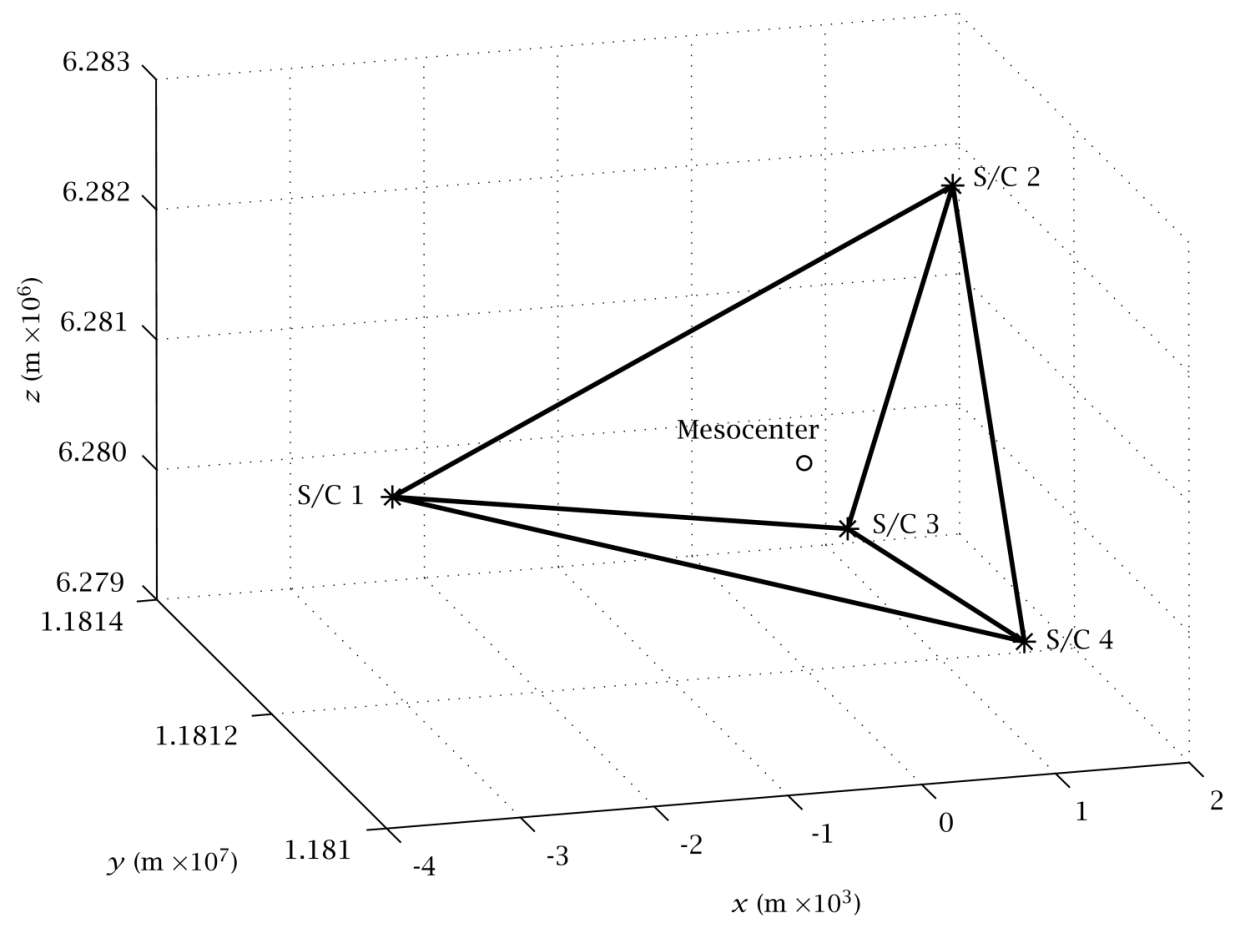

FIG. 3. Three-Dimensional View of Optimal Terminal Tetrahedron for Two-Maneuver Problem. 
TABLE 1. Maneuver Durations for the Two-Maneuver Problem

\begin{tabular}{|c|c|c|c|c|}
\hline Burn Duration(s)\Spacecraft Number & 1 & 2 & 3 & 4 \\
\hline First Burn & 65.1965 & 65.2958 & 65.2960 & 65.1584 \\
\hline Second Burn & 0 & 0 & 0 & 0.0356 \\
\hline
\end{tabular}

Notice in Table 1 that, despite the presence of two maneuver opportunities, only one spacecraft actually thrusts twice. Three of the four spacecraft are able to complete the transfer and maintain equal semimajor axes with only the first maneuver. The fourth spacecraft, however, uses a slight corrective maneuver later in the orbit, at a true anomaly of approximately 90 degrees. Interestingly, as seen in Fig. 4, the fourth spacecraft is also the spacecraft with the lowest final altitude. The small second maneuver is predominantly in the tangential direction (see Fig. 5) and raises the semimajor axis of that spacecraft to match the other three. With such a small second maneuver, one wonders if this maneuver is even necessary. We will show in the next section that this problem can be solved with only one maneuver allowed per spacecraft, but the one-maneuver solution used $0.15 \mathrm{~kg}$ more fuel than the two-maneuver trajectory. Further discussion comparing the two scenarios is presented in the next subsection.

As seen in Fig. 6, the optimal locations of the first maneuvers for each of the spacecraft are near the reference perigee, resembling a Hohmann transfer. Upon closer inspection of Fig. 6, it is seen that all four burns are offset by varying amounts from the reference perigee. By staggering the burns, the three spacecraft

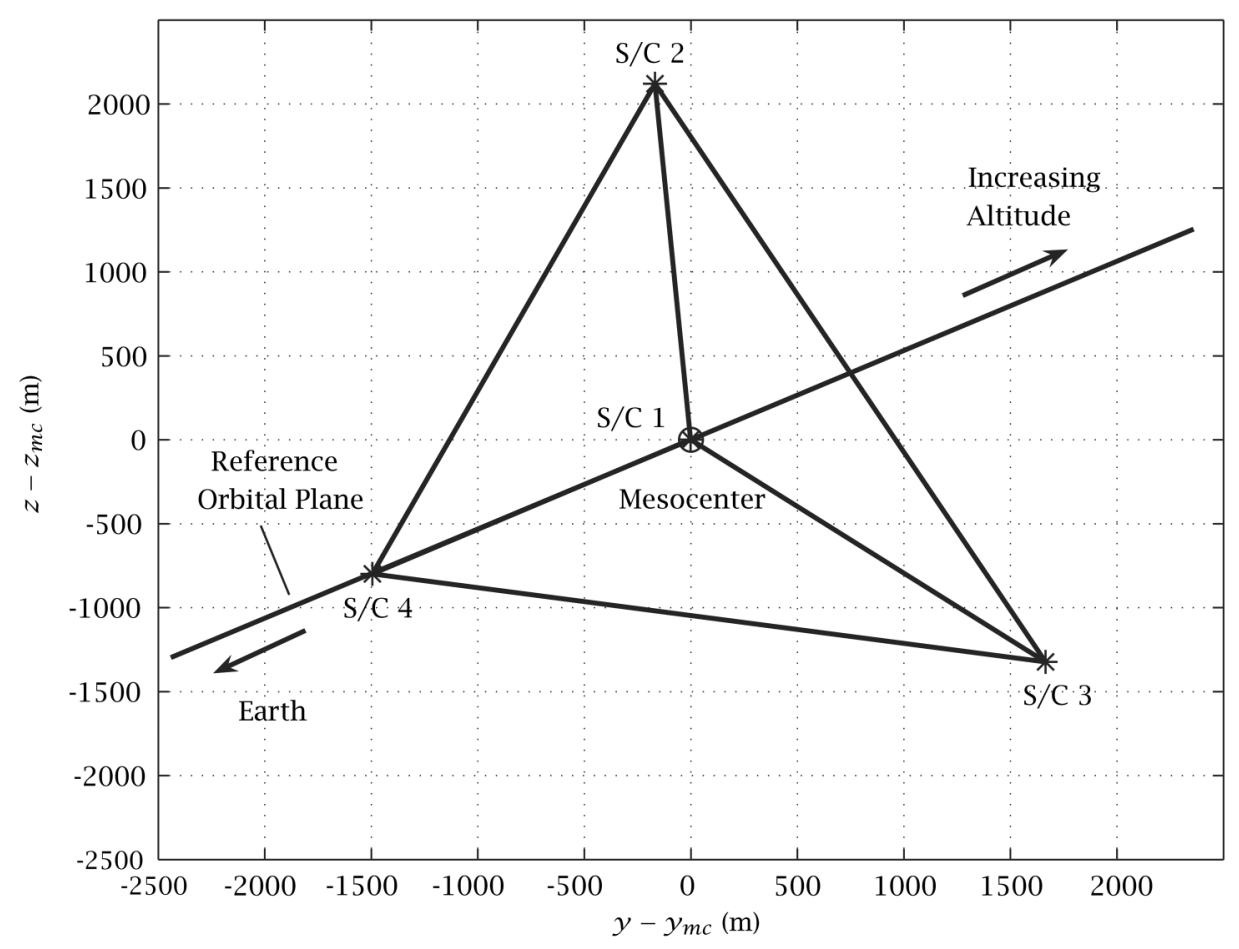

FIG. 4. Optimal Terminal Tetrahedron Viewed Along Orbital Plane for Two-Maneuver Problem. 


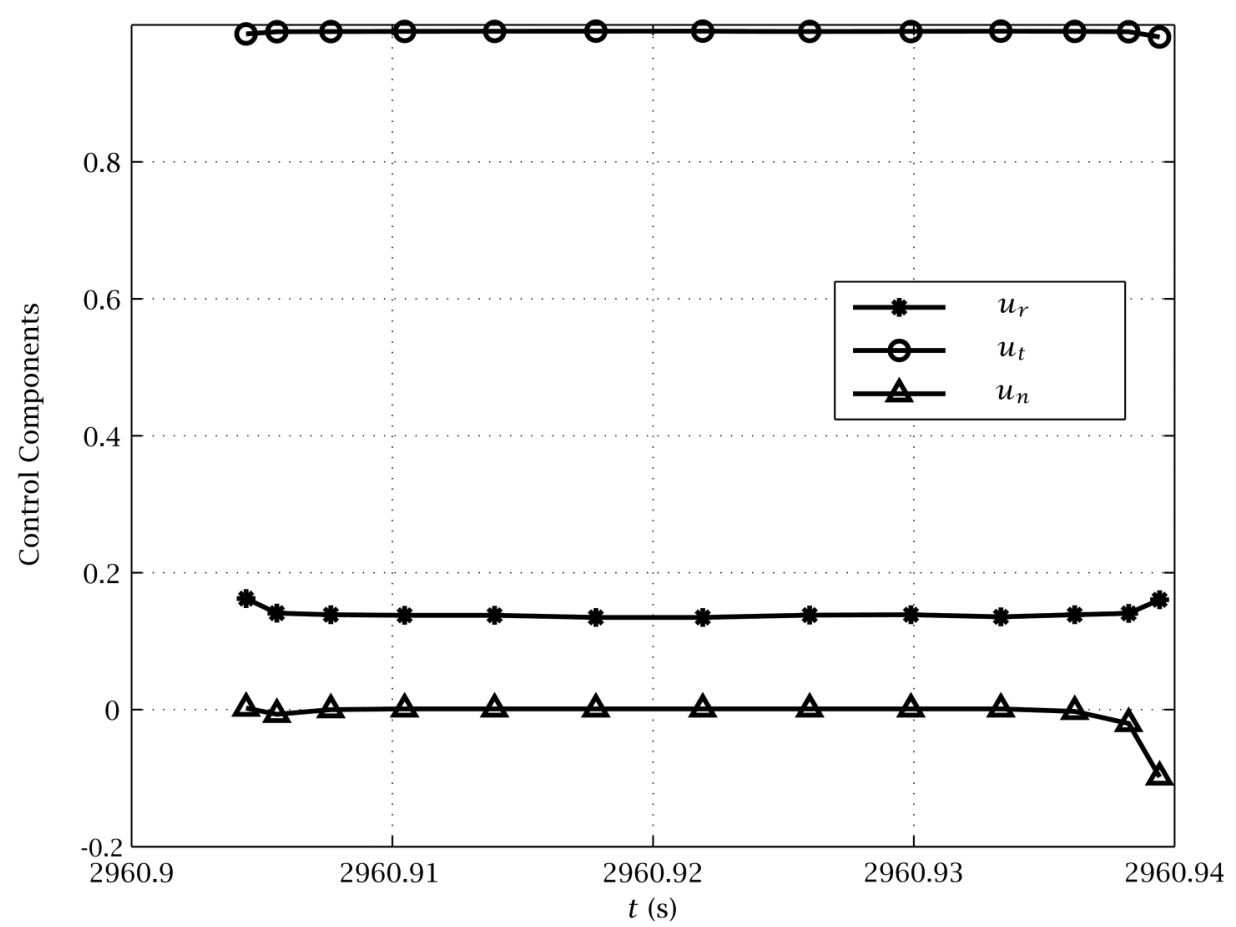

FIG. 5. SC \#4 Control for the Second Maneuver in the Two-Maneuver Problem.

reach the desired geometric positions but attain the same semimajor axis upon completion of the maneuver. These maneuvers do use slightly different amounts of fuel, and when comparing Fig. 7 and Fig. 4 it is seen that the amount of fuel burned by each spacecraft near perigee increases with increasing terminal altitude.

One interesting aspect of the formation is the discovery of the fuel-optimal orientation of the tetrahedron. Recall that no constraints are placed on the orientation of the formation, yet one can see a definite structure to the solution by looking at Figs. 4 and 8. In Fig. 4, it is seen that two of the spacecraft remain in the orbit plane, while the other two spacecraft are located symmetrically out of the orbit plane. Figure 8 shows the view of the formation from a direction normal to the orbit plane. In particular, Fig. 8 shows the terminal position of each spacecraft relative to the mesocenter where $x_{r, m c}, x_{\theta, m c}, x_{n, m c}$ represent the instantaneous radial, transverse, and normal components of the position of the mesocenter. It is seen from Fig. 8 that the two out-of-plane spacecraft ( 2 and 3 ) have the same projection into the orbit plane. Furthermore, the altitude of Spacecraft 4, which burns twice, is significantly lower than the altitude of the other three spacecraft. Although the solution is not guaranteed to be globally optimal, it was found that several different initial guesses all converged to the solution obtained in this analysis.

Figures 9-11 show the maneuver profile for the three components of thrust for all four spacecraft during the first maneuver. The four arcs in each figure represent the four spacecraft. Note that Figs. 9-11 include the control at the boundaries, which was calculated using the HBVP conditions of equations (44) and (45). The overwhelming majority of the thrust is in the transverse direction (Fig. 10), due to the orbit transfer. Consistent with the relative geometry in Fig. 4, only two spacecraft 


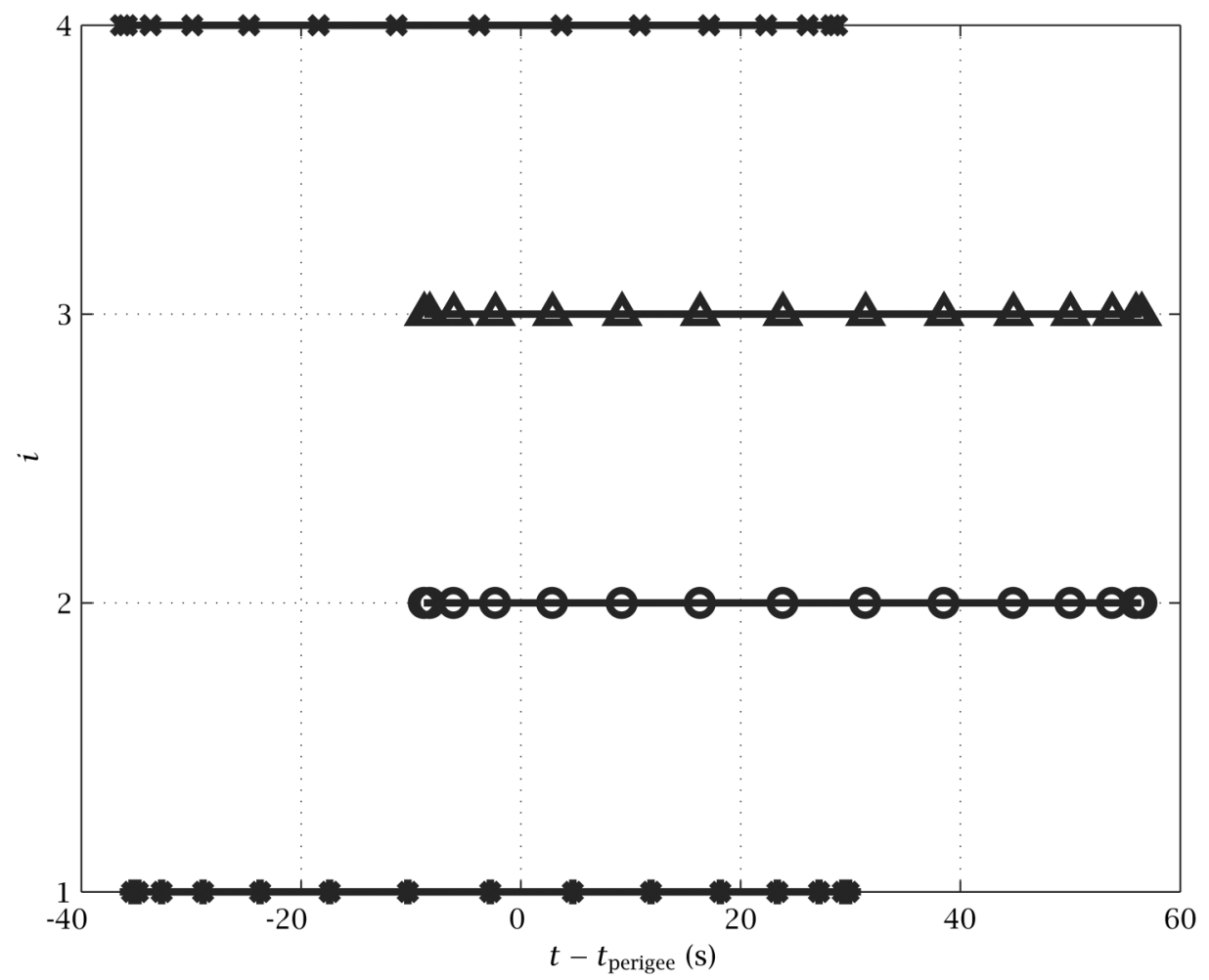

FIG. 6. Spacecraft Burn Durations Relative to Time of Reference Perigee Passage, $t-t_{\text {perigee }}$, for Spacecraft $i=1, \ldots, 4$ During First Burn Phase for Two-Maneuver Problem.

have nonzero normal components of thrust (Fig. 11), since two spacecraft remain in the reference orbit plane. Interestingly, the small radial component of thrust in all four spacecraft (Fig. 9) is negative at the start of the maneuver and positive at the end of the maneuver. By examining Gauss's variational equations [35], we see that a change in semimajor axis, $a$, is related to the radial acceleration, $a_{r}$, by

$$
\dot{a}=\frac{2 a^{2}}{h}\left(e \sin (v) a_{r}+\frac{p}{r} a_{\theta}\right)
$$

where $v$ is the true anomaly, $h$ is the angular momentum, $p$ is the semilatus rectum, $e$ is the eccentricity, and $r$ is the radial distance to the spacecraft. From equation (46) it is seen that when $v$ is slightly negative (i.e., just before perigee), the radial acceleration must be negative in order to increase the semimajor axis. Similarly, when $v$ is slightly positive (i.e., just after perigee), the radial acceleration must be positive to increase the semimajor axis. Therefore starting the maneuver in the negative radial direction before perigee aids in raising the semimajor axis of the spacecraft. Note that the crossover point for each spacecraft is not exactly at perigee, but this slight difference is most likely attributed to the need to satisfy the terminal conditions.

\section{Single-Maneuver Solution}

In the two-maneuver solution, only one of the four spacecraft used both maneuver opportunities. Moreover, the spacecraft that did use two maneuvers had an 


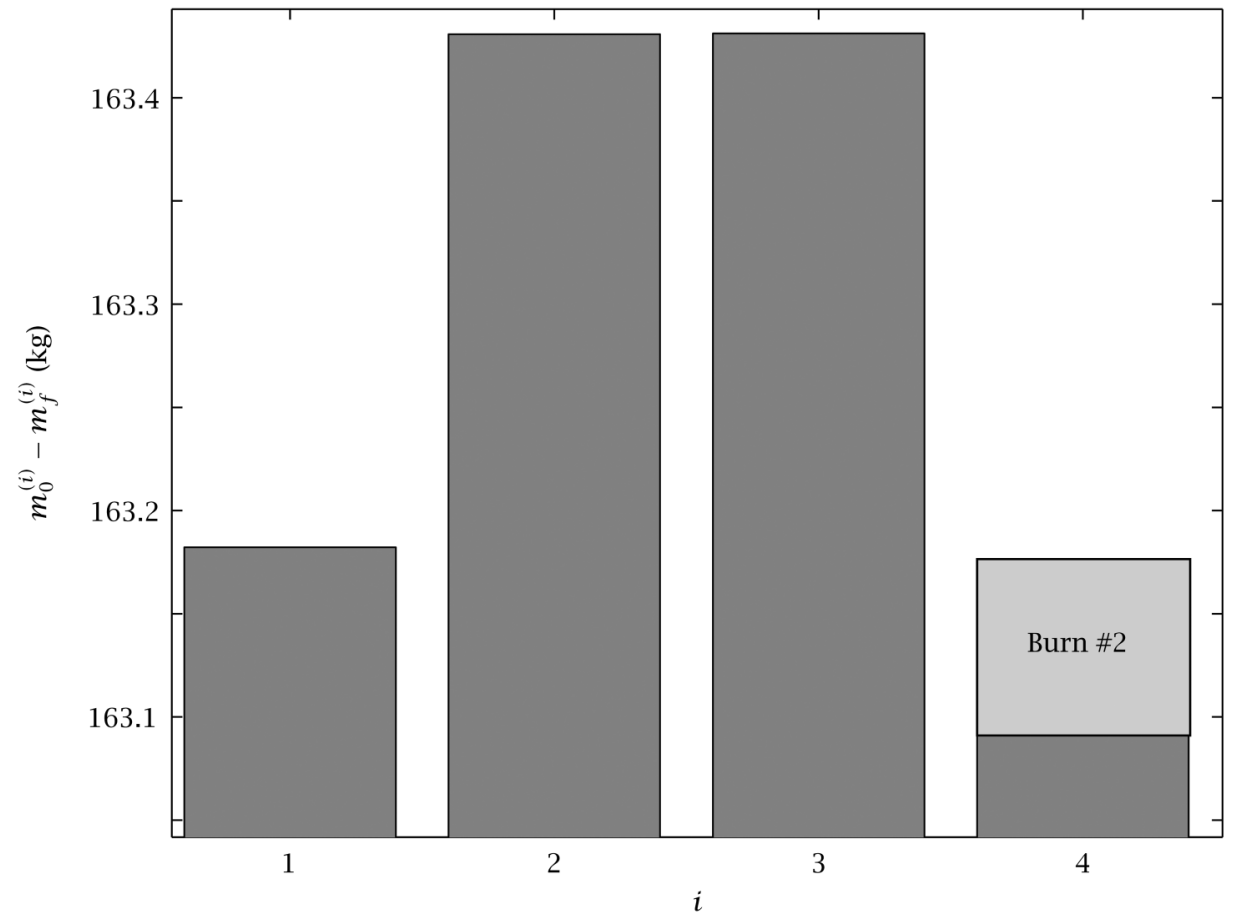

FIG. 7. Optimal Fuel Consumption $m_{0}^{(i)}-m_{f}^{(i)}$ of Spacecraft $i=1, \ldots, 4$ for Two-Maneuver Problem.

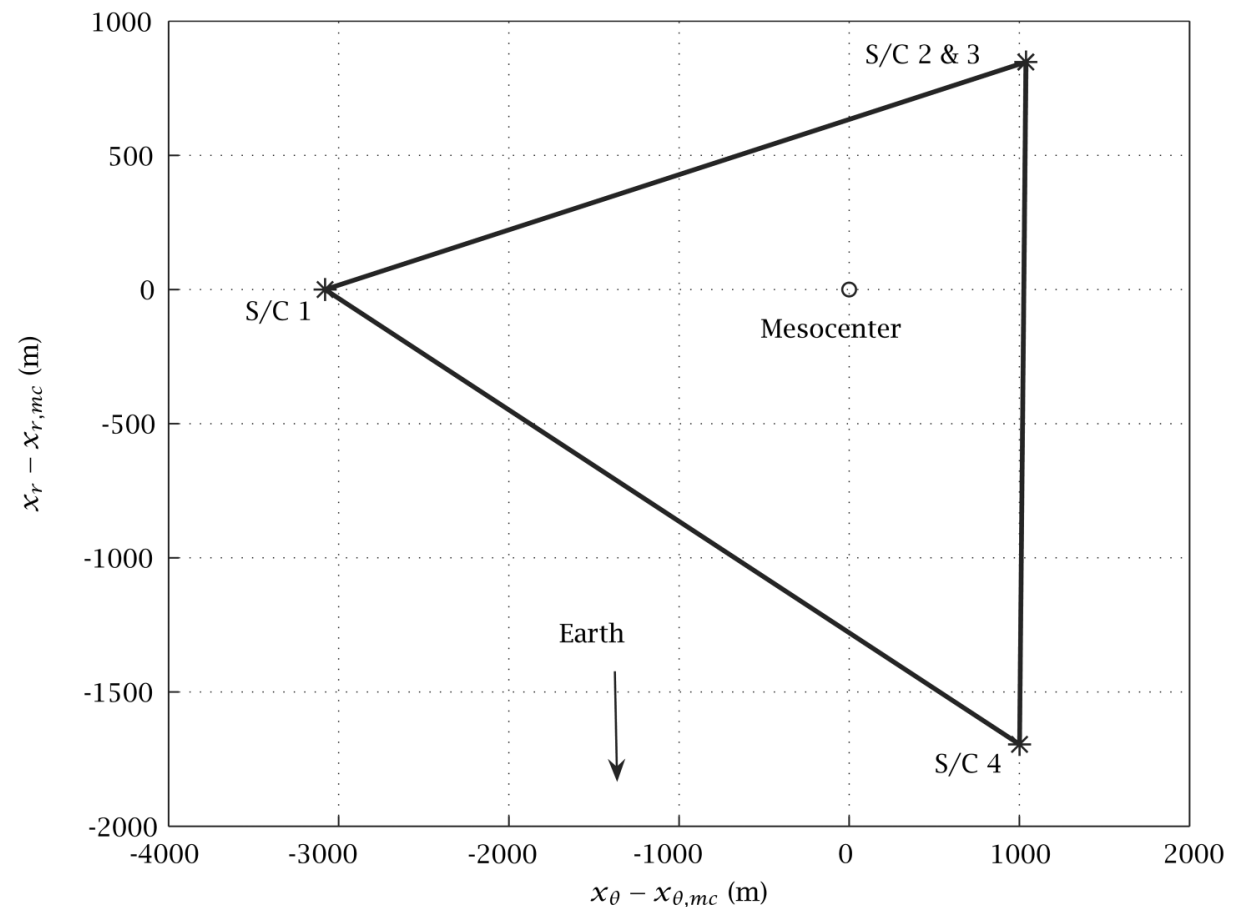

FIG. 8. Optimal Terminal Tetrahedron Viewed from Normal to the Orbital Plane for Two-Maneuver Problem. 


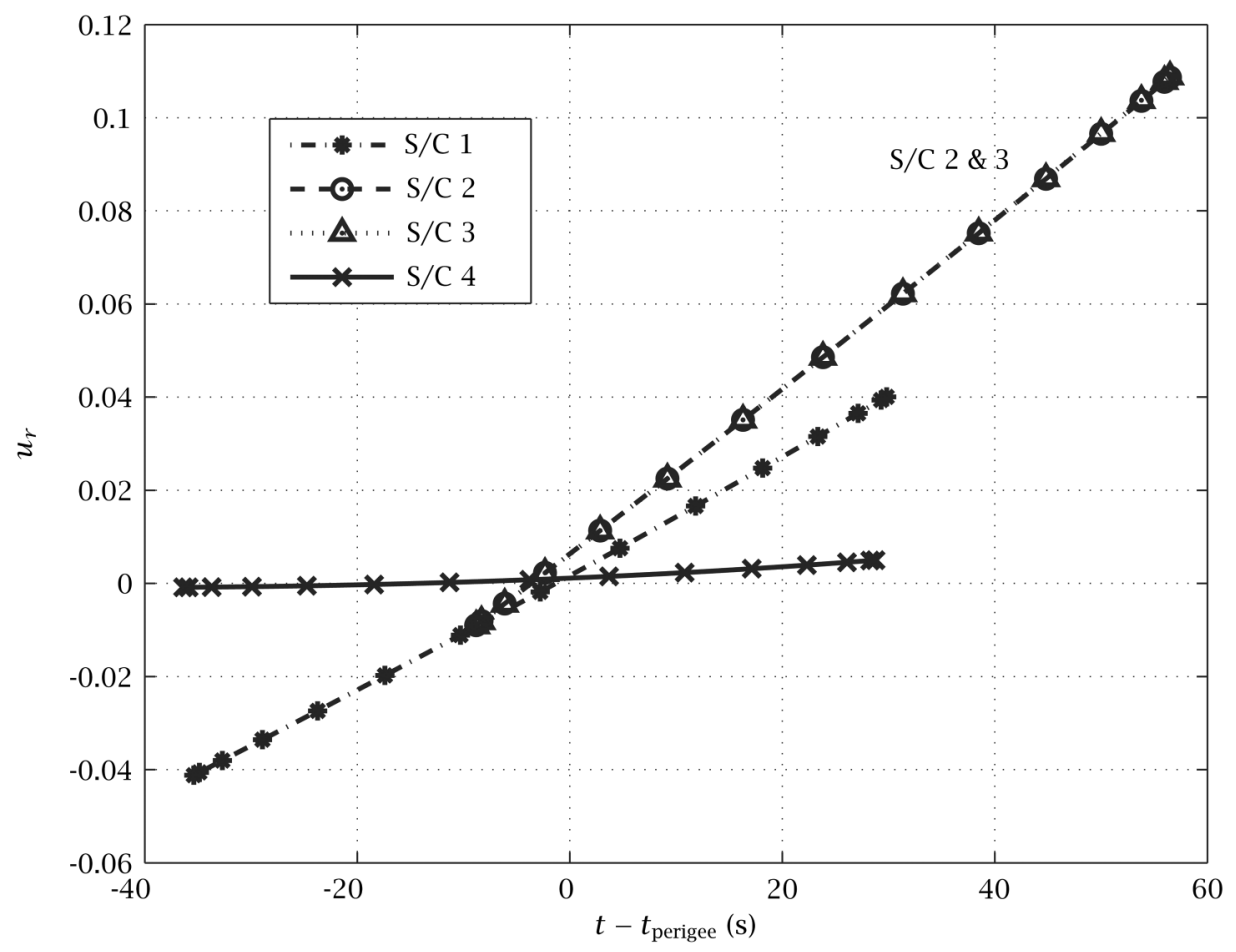

FIG. 9. Radial Control for the Two-Maneuver Problem.

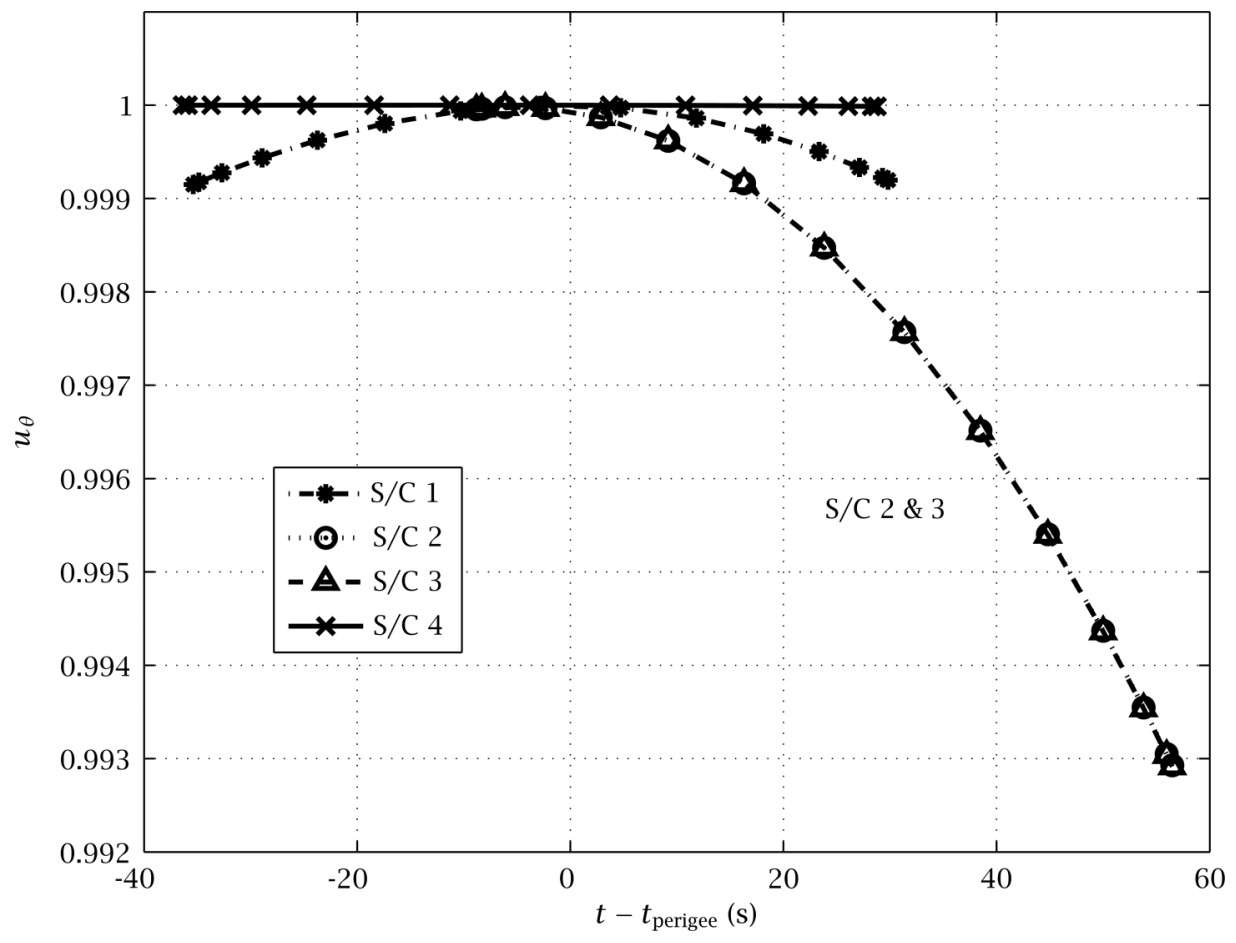

FIG. 10. Transverse Control for the Two-Maneuver Problem. 


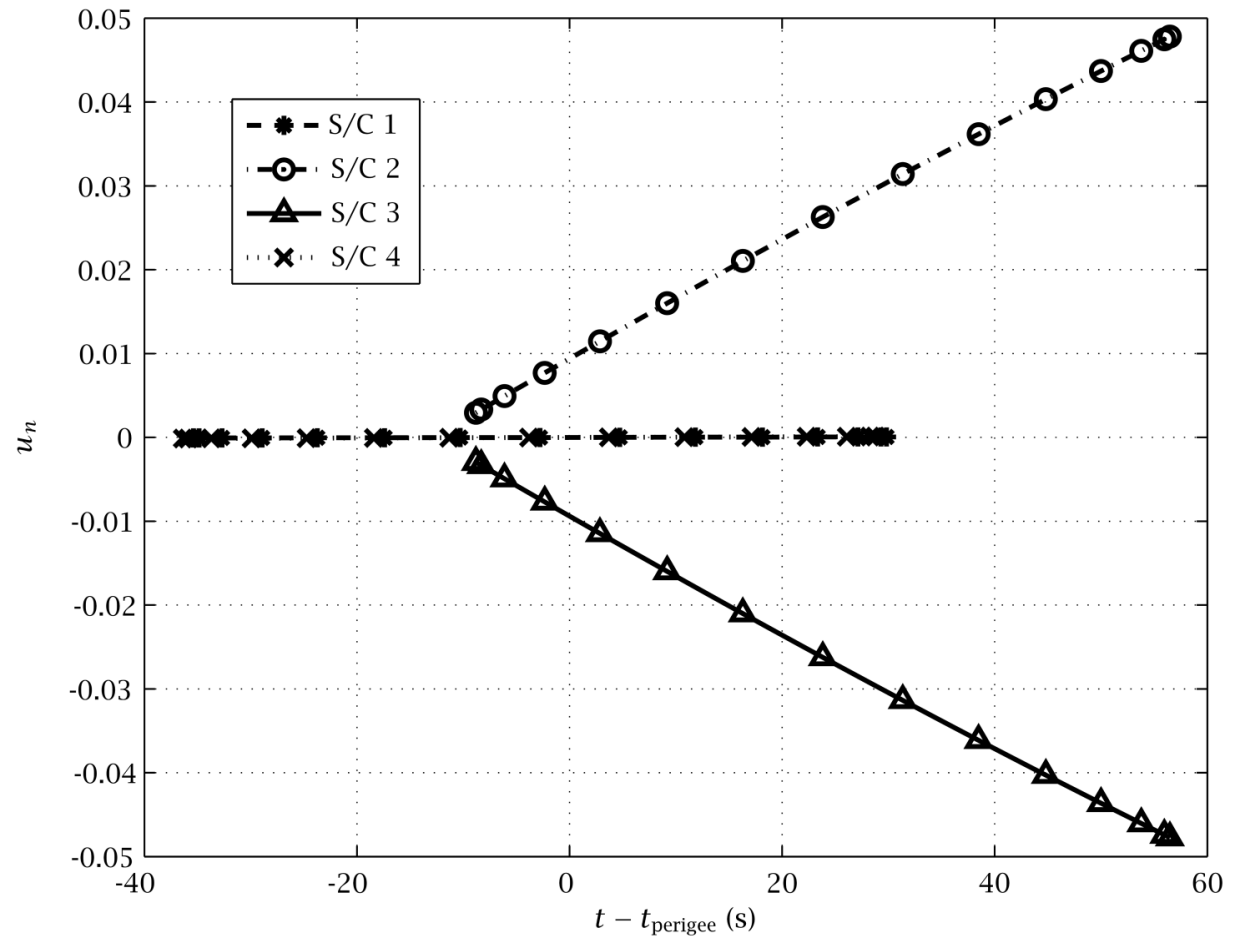

FIG. 11. Normal Control for the Two-Maneuver Problem.

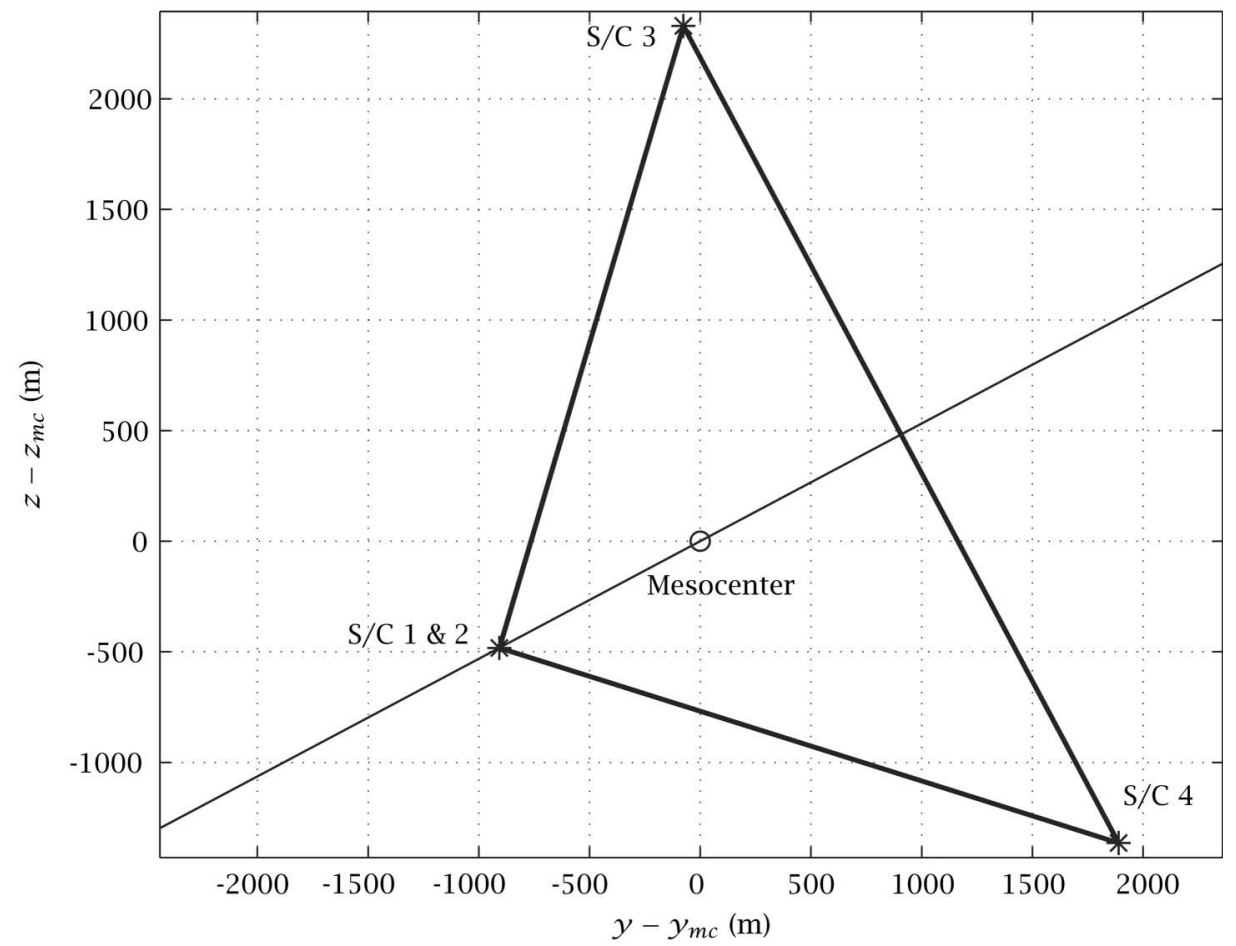

FIG. 12. Optimal Terminal Tetrahedron Viewed Along Orbital Plane for Single-Maneuver Problem. 


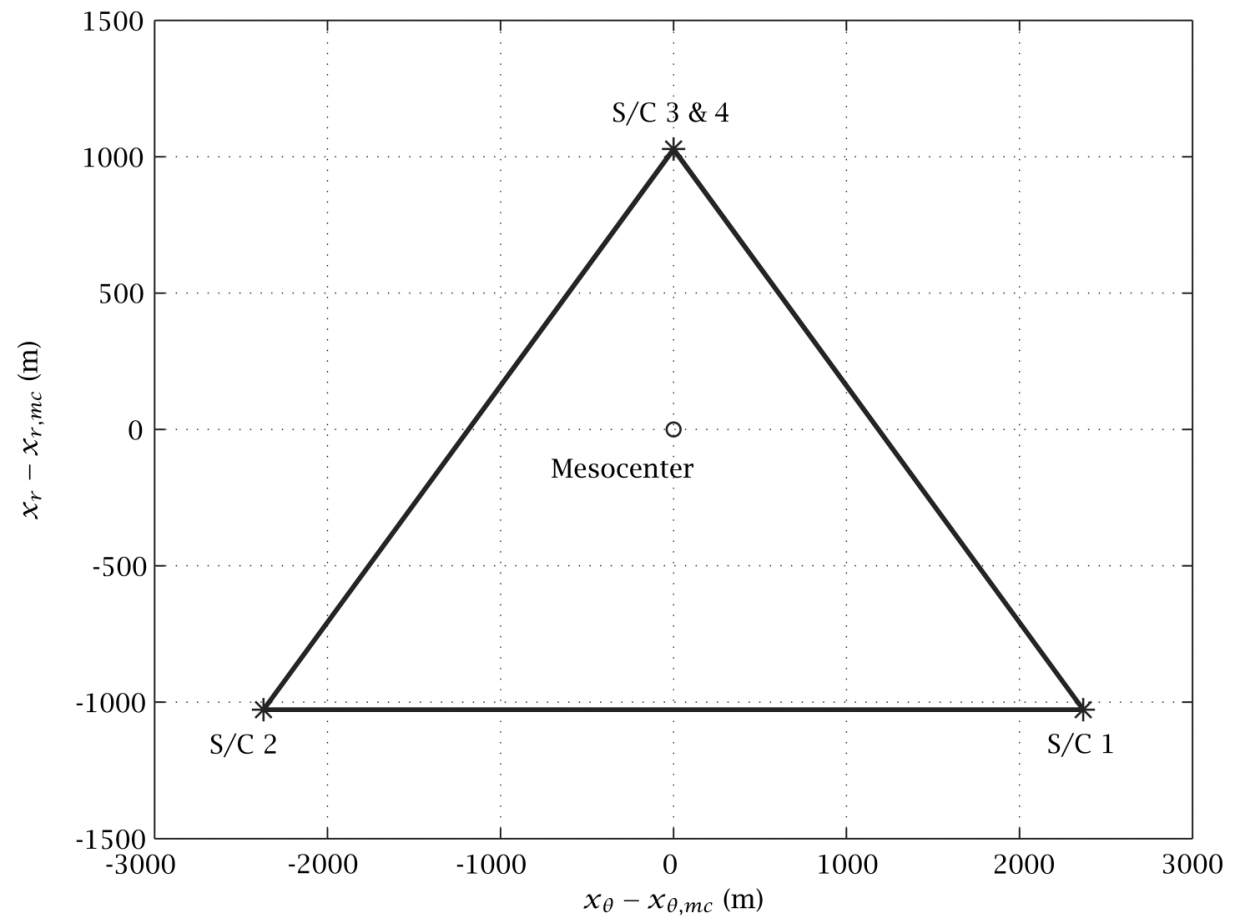

FIG. 13. Optimal Terminal Tetrahedron Viewed from Normal to the Orbital Plane for Single-Maneuver Problem.

extremely small second maneuver. A natural question that arises from the twomaneuver result is what the solution would be when each spacecraft is only allowed one maneuver opportunity. The single-maneuver problem was posed by setting the duration of the second burn to zero.

As expected, slightly more fuel is used in the single-maneuver solution: $653.375 \mathrm{~kg}$ as opposed to $653.220 \mathrm{~kg}$ in the two-maneuver solution. Interestingly, allowing only one maneuver per spacecraft changes the orientation of the tetrahedron at the terminal time. The orientation of the tetrahedron for the single-maneuver solution is seen in Figs. 12 and 13. By allowing only one maneuver per spacecraft, the single burn must simultaneously achieve two conflicting goals. On one hand, at least one spacecraft must terminate at a different altitude than the other three in order to satisfy the tetrahedral constraints. With the periodicity constraint, the only way to achieve a different altitude at the final time is by staggering the maneuvers to occur at different times. On the other hand, each spacecraft should burn at perigee in order to effciently complete the orbit transfer. To minimize fuel, each spacecraft should burn as close as possible to perigee, yet still be staggered enough to produce sufficient separation in the final geometry. By balancing these two goals, the resulting effect is to minimize the maximum altitude separation. This result can be seen in Fig. 13, where the difference between all four spacecraft and the altitude of the mesocenter is the same. Furthermore, from Fig. 14, it can be seen that the spacecraft must be staggered further away from perigee than in the two-maneuver solution, which is the likely cause of the larger fuel expenditure than the two-burn solution. Two of the spacecraft burn almost completely before perigee while the other two are 


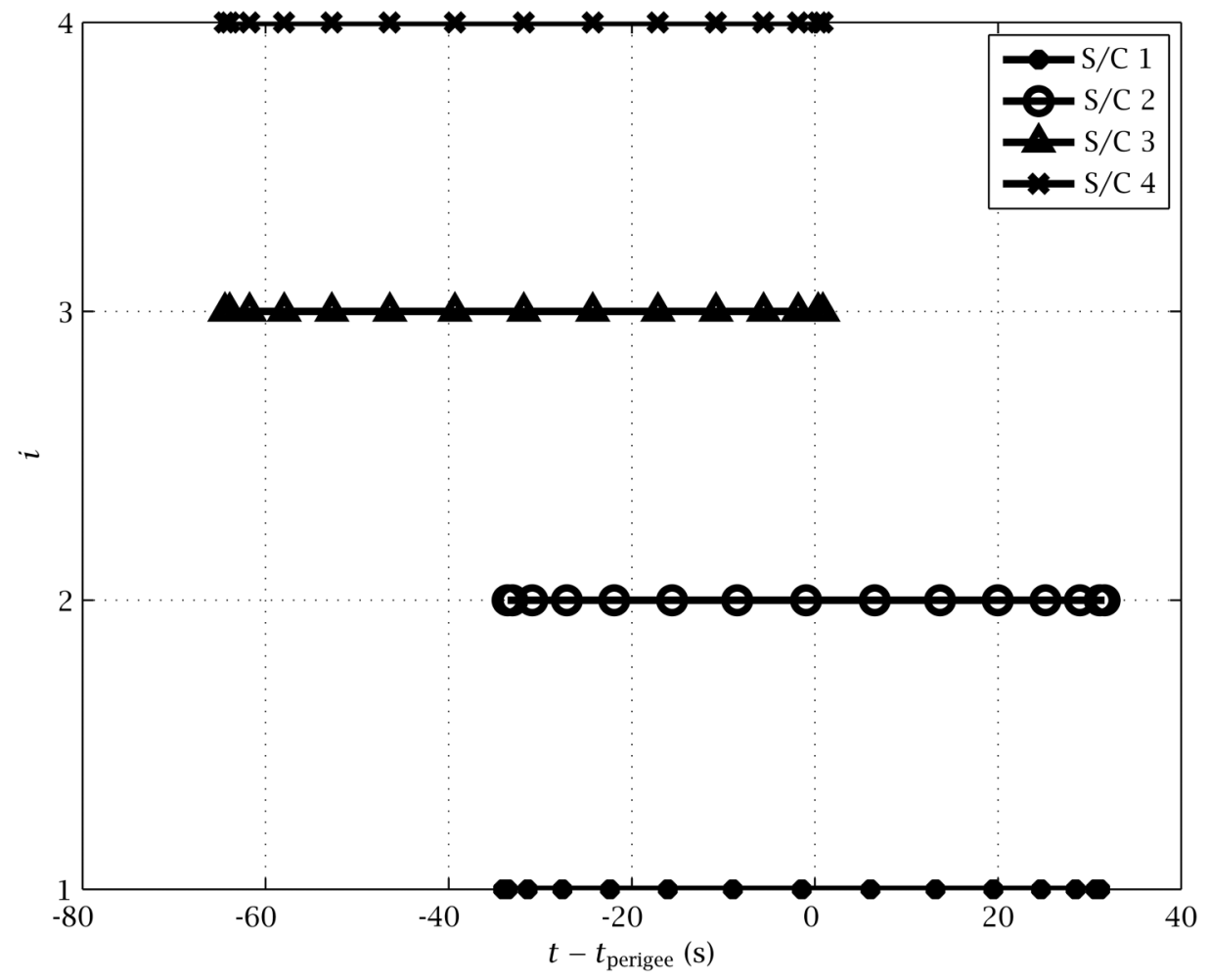

FIG. 14. Spacecraft Burn Durations Relative to Time of Reference Perigee Passage, $t-t_{\text {perigee }}$, for Spacecraft $i=1, \ldots, 4$ During First Burn Phase for the Single-Maneuver Problem.

approximately centered around perigee. From a fuel balancing perspective, the difference in expended fuel between the four spacecraft is $0.25 \mathrm{~kg}$. In fact, the difference in expended fuel between the four spacecraft for the two-burn solution is also $0.25 \mathrm{~kg}$, (seen in Fig. 7), implying that there is no significant advantage in choosing one scenario over the other with respect to fuel balancing.

\section{Analysis of Optimality for the Single-Maneuver Problem}

As mentioned previously, a primary benefit of the Gauss Pseudospectral Method is the equivalence that exists between the direct and indirect approaches [30]. By using the costate mapping shown in this paper, one can verify that the control from the NLP matches the analytic control determined from the first-order optimality conditions at the LG points. By verifying this equivalence, one can gain more confidence that the solution obtained from the NLP is an extremal solution of the optimal control problem.

This analysis was done for both the single-maneuver and two-maneuver cases, but only the results from the single-maneuver case are shown here. Figures 15-17 show the magnitude of the differences between the NLP control and the estimated optimal control derived from the HBVP conditions for the three components of control, $\left|\Delta u_{r}\right|,\left|\Delta u_{\theta}\right|$, and $\left|\Delta u_{n}\right|$. It is seen from Figs. 15-17 that the largest magnitude difference is of order $10^{-6}$, verifying that the NLP control is in excellent agreement with the estimated control from the first-order optimality conditions. This error can potentially be reduced further by increasing the number of LG points or 


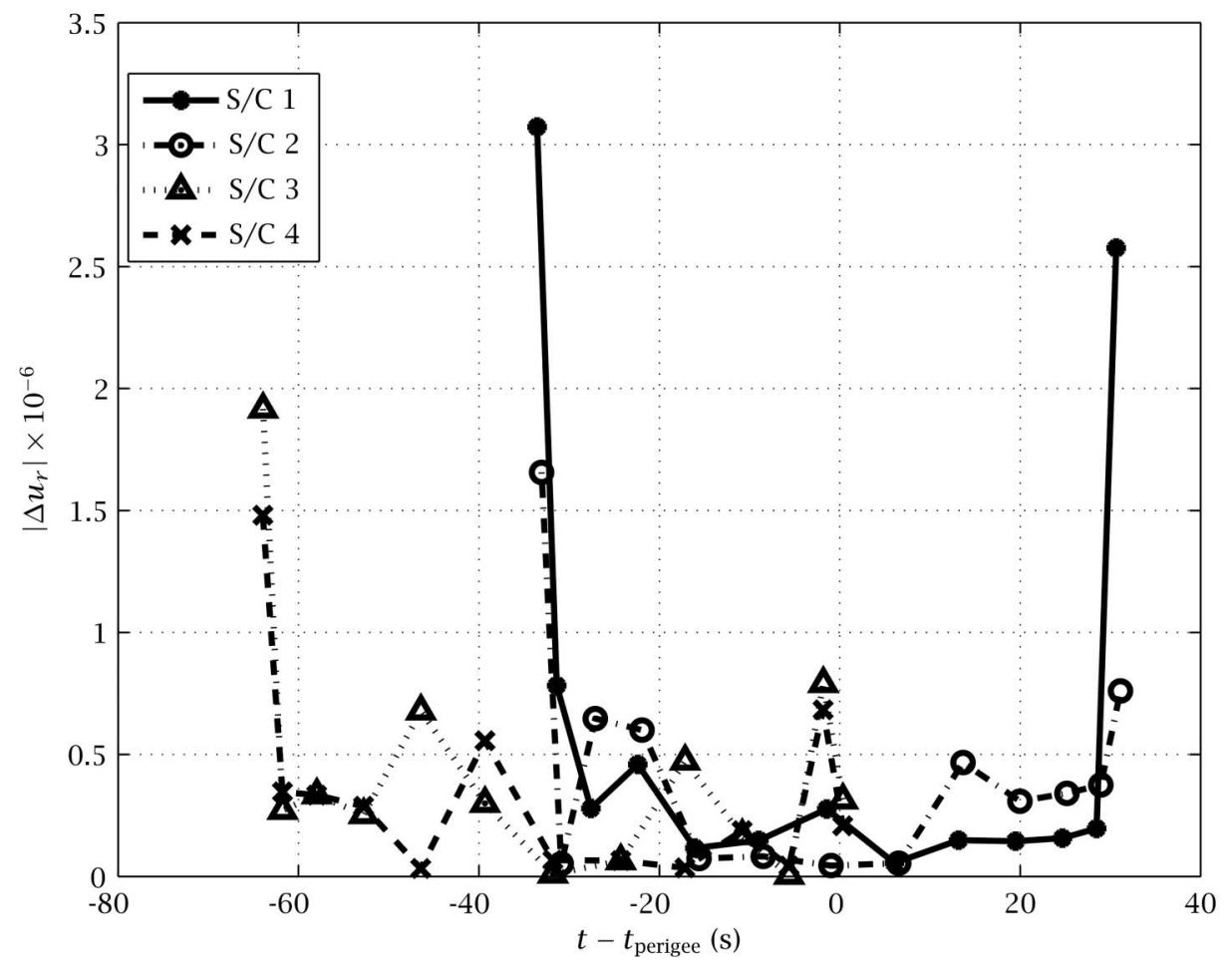

FIG. 15. Error in Radial Control for the Single-Maneuver Problem.

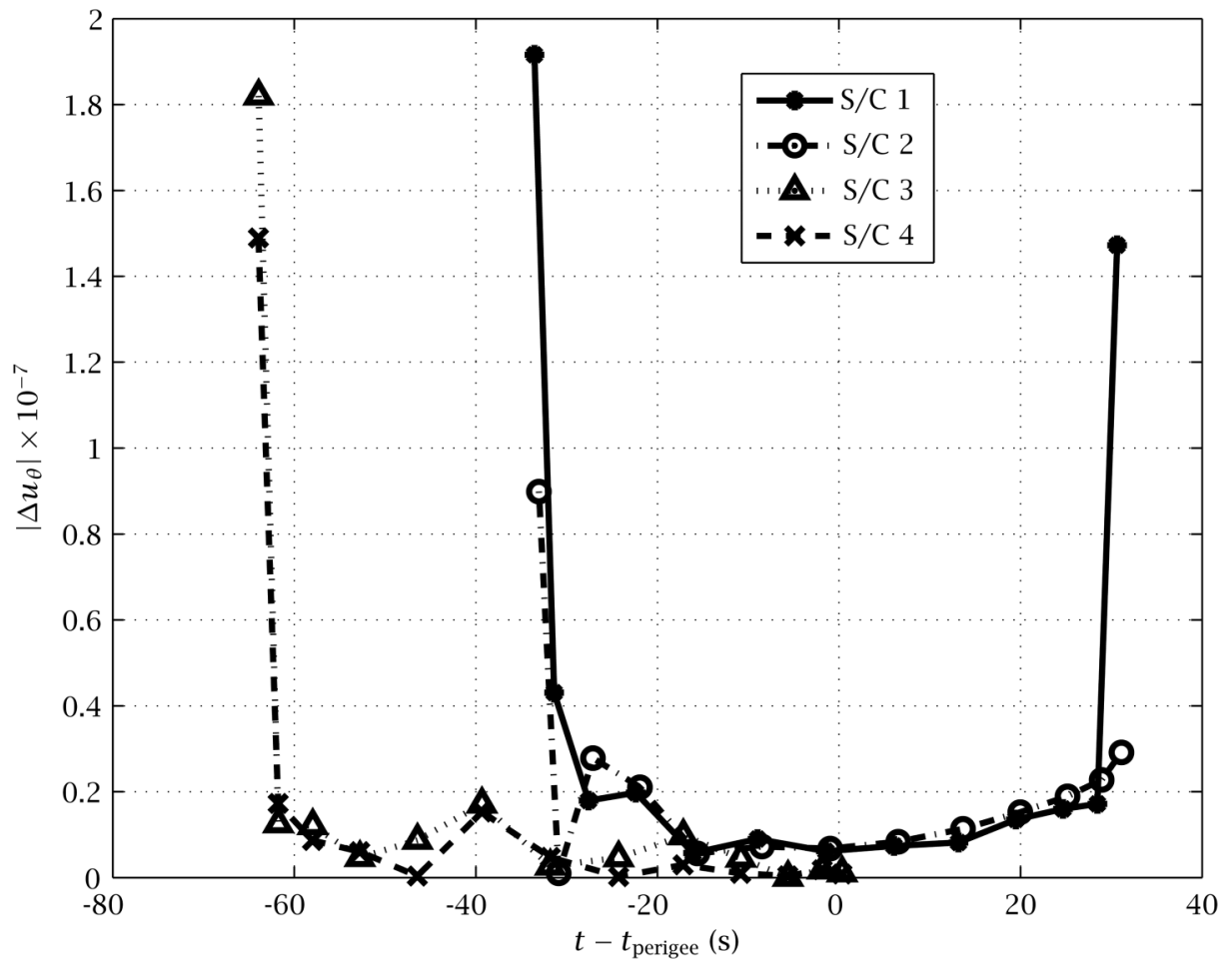

FIG. 16. Error in Transverse Control for the Single-Maneuver Problem. 


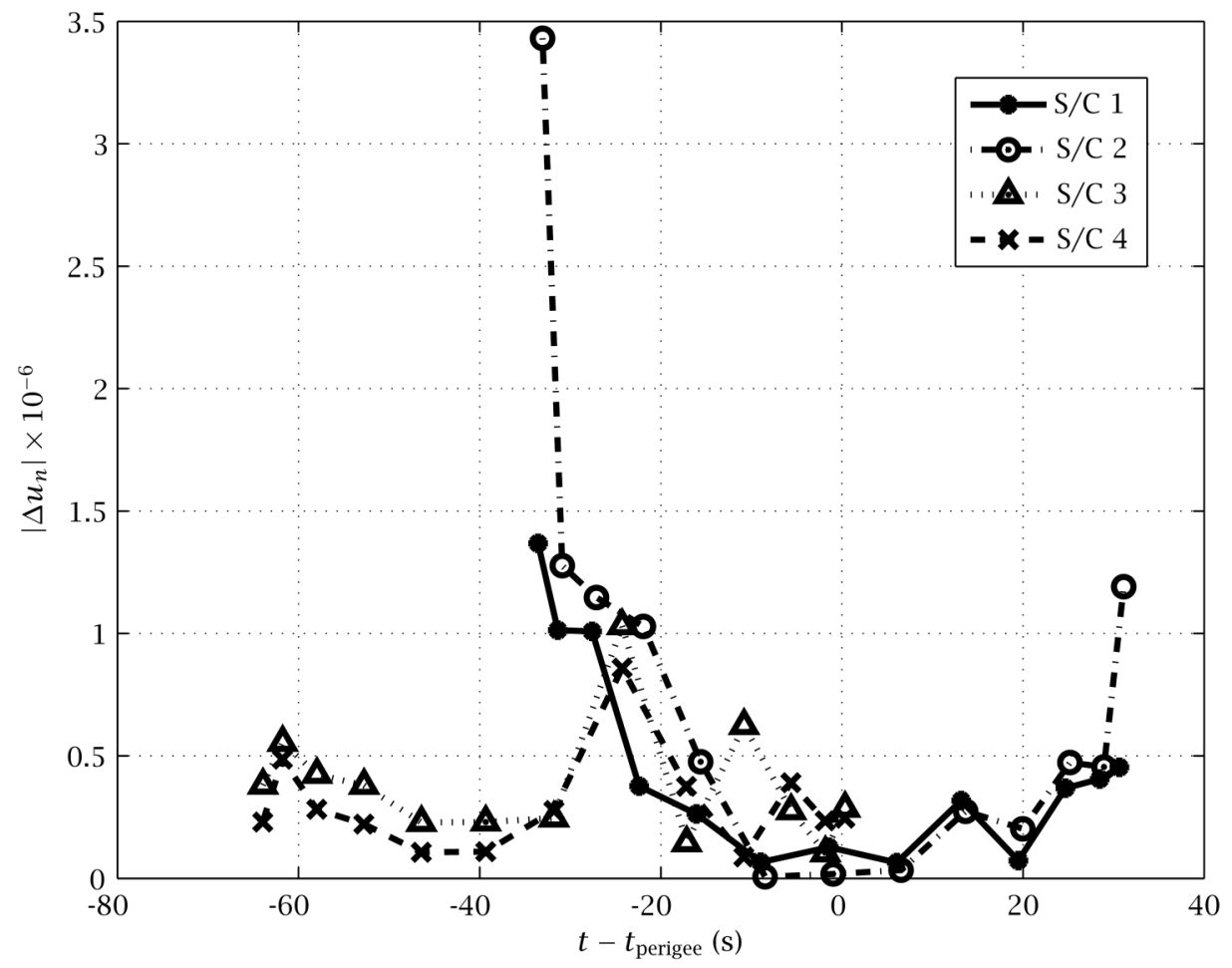

FIG. 17. Error in Normal Control for the Single-Maneuver Problem.

by tightening the tolerances of the NLP optimizer. Recall that there are no control variables in the NLP at the boundaries, and therefore only the control at the LG points are compared.

\section{Conclusions}

The problem of determining minimum-fuel maneuver sequences for a fourspacecraft formation was considered. The objective was to determine fuel-optimal configuration trajectories that would transfer a four spacecraft formation from an initial parking orbit to a desired terminal reference orbit while satisfying particular tetrahedral formation constraints. The configuration problem was solved numerically using a newly developed direct transcription method called the Gauss pseudospectral method. In particular, two versions of the minimum-fuel configuration problem were considered. In the first scenario each spacecraft was given two maneuver opportunities to complete the transfer. In the second scenario each spacecraft was given only one maneuver opportunity. Optimal solutions, and the discovery of the fuel-optimal orientation, were found for each scenario. In addition, the first-order optimality conditions (or KKT conditions) obtained from the Gauss pseudospectral method were shown for a multiple-phase optimal control problem. These KKT conditions were compared with the optimality conditions obtained by discretizing the calculus of variations problem via the Gauss pseudospectral method. A mapping between the KKT multipliers and the HBVP costates was used to determine an estimate of the optimal control for a four spacecraft formation flying problem. It was found that the control obtained from the NLP was in excellent 
agreement with the control estimated by the discretized HBVP. The results of this research provide insight into the structure of formation configuration trajectories and demonstrate the advantages of using the Gauss pseudospectral method for formation flying trajectory design.

\section{Disclaimer}

Any opinions, findings, and conclusions or recommendations expressed in this material are those of the authors and do not necessarily reflect the views of the National Aeronautics and Space Administration.

\section{Acknowledgments}

This work was funded by the NASA Goddard Spaceflight Center under Cooperative Agreement NAS5-NCC5-730. The authors would like to thank Mr. Stephen P. Hughes as technical monitor for his insights and helpful suggestions during the course of this work.

\section{References}

[1] SCHARF, D. P., HADAEGH, F. Y., and PLOEN, S. R. "A Survey of Spacecraft Formation Flying Guidance and Control (Part II): Control," Proceedings of the American Control Conference, Boston, MA, 2004, pp. 2976-2985.

[2] SCHARF, D. P., HADAEGH, F. Y., and PLOEN, S. R. "A Survey of Spacecraft Formation Flying Guidance and Control (Part I): Guidance," Proceedings of the American Control Conference, Denver, CO, 2003, pp. 1733-1739.

[3] MAILHE, L. M. and GUZMAN, J. J. "Initialization and Resizing of Formation Flying using Global and Local Optimization Methods," Proceedings of the 2004 IEEE Aerospace Conference, Vol. 1, March 6-13, 2004, p. 556.

[4] HOSKINS, A. and ATKINS, E. "Spacecraft Formation Optimization with a Multi-Impulse Design," Proceedings of the 2005 AIAA Guidance, Navigation, and Control Conference, AIAA Paper 2005-5835, August 15-18, 2005, San Francisco, CA.

[5] ZANON, D. and CAMPBELL, M. "Mission Objectives in Tetrahedral Formation Maneuvering," Proceedings of the 2005 AIAA Guidance, Navigation, and Control Conference, AIAA Paper 2005-5834, August 15-18, 2005, San Francisco, CA.

[6] GUZMAN, J. J. "Tetrahedron Formation Control," The Journal of the Astronautical Sciences, Vol. 51, No. 4, 2003, pp. 419-431.

[7] TSUDA, Y. "Global Optimization of Maneuver Schedule for Multiple Spacecrafts Flying in Formation," presented as paper IAC-04-A.2.01 at the 55th International Astronautical Congress, Vancouver, Canada, 2004.

[8] BETTS, J. T. "Survey of Numerical Methods for Trajectory Optimization," Journal of Guidance, Control, and Dynamics, Vol. 21, No. 2, March-April 1998, pp. 193-207.

[9] ATHANS, M. A. and FALB, P. L. Optimal Control, McGraw-Hill, New York, 1966.

[10] BETTS, J. T. Practical Methods for Optimal Control Using Nonlinear Programming, Society for Industrial and Applied Mathematics Press, 2001.

[11] CANUTO, C., HUSSAINI, M. Y., QUARTERONI, A., and ZANG, T. A. Spectral Methods in Fluid Dynamics, Springer-Verlag, New York, 1988.

[12] ELNAGAR, G., KAZEMI, M., and RAZZAGHI, M. "The Pseudospectral Legendre Method for Discretizing Optimal Control Problems," IEEE Transactions on Automatic Control, Vol. 40, No. 10, October 1995.

[13] FAHROO, F. and ROSS, I. M. "Costate Estimation by a Legendre Pseudospectral Method," Journal of Guidance, Control, and Dynamics, Vol. 24, No. 2, March-April 2002, pp. 270-277.

[14] FAHROO, F. and ROSS, I. M. "Direct Trajectory Optimization by a Chebyshev Pseudospectral Method," Journal of Guidance, Control, and Dynamics, Vol. 25, No. 1, January-February 2002, pp. $160-166$.

[15] FORNBERG, B. A Practical Guide to Pseudospectral Methods, Cambridge University Press, 1998.

[16] ELNAGAR, G.E. and KAZEMI, M. A. "Pseudospectral Legendre-Based Optimal Computation of Nonlinear Constrained Variational Problems," Journal of Computational and Applied Mathematics, Vol. 88, 1997, pp. 363-375. 
[17] CUTHRELL, J.E. and BIEGLER, L. T. "On the Optimization of Differential-Algebraic Process Systems," AIChE Journal, Vol. 33, No. 8, August 1987, pp. 1257-1270.

[18] CUTHRELL, J. E. and BIEGLER, L. T. "Simultaneous Optimization and Solution Methods for Batch Reactor Control Profiles," Computers and Chemical Engineering, Vol. 13, No. 1-2, 1989, pp. 49-62.

[19] REDDIEN, G. W. “Collocation at Gauss Points as a Discretization in Optimal Control," SIAM Journal of Control and Optimization, Vol. 17, No. 2, March 1979.

[20] TREFETHEN, L. N. Spectral Methods in MATLAB, SIAM Press, Philadelphia, 2000.

[21] FAHROO, F. and ROSS, I. M. "A Spectral Patching Method for Direct Trajectory Optimization," The Journal of the Astronautical Sciences, Vol. 48, No. 2-3, April-September 2000, pp. 269-286.

[22] ROSS, I. M. and FAHROO, F. "A Direct Method for Solving Nonsmooth Optimal Control Problems," Proceedings of the 2002 World Congress of the International Federation on Automatic Control, IIAC, Barcelona, July 2002.

[23] RAO, A. V. "Extension of a Pseudospectral Legendre Method to Non-Sequential MultiplePhase Optimal Control Problems," presented as paper AIAA 2003-5634 at the AIAA Guidance, Navigation, and Control Conference, Austin, TX, August 11-14, 2003.

[24] ROSS, I. M. and FAHROO, F. "Pseudospectral Knotting Methods for Solving Optimal Control Problems," Journal of Guidance, Control, and Dynamics, Vol. 27, No. 3, May-June 2004, pp. 397-405.

[25] ELNAGAR, G. and KAZEMI, M. "Pseudospectral Chebyshev Optimal Control of Constrained Nonlinear Dynamical Systems," Computational Optimization and Applications, Vol. 11, 1998, pp. 195-217.

[26] ROSS, I. M. and FAHROO, F. "A Pseudospectral Transformation of the Covectors of Optimal Control Systems," Proceedings of the 1st IFAC/IEEE Symposium on Structure and Control, Prague, Czech Republic, August 2001.

[27] ROSS, I. M. and FAHROO, F. "Legendre Pseudospectral Approximations of Optimal Control Problems," Lecture Notes in Control and Information Sciences, Springer-Verlag, 2003.

[28] BENSON, D. A. A Gauss Pseudospectral Transcription for Optimal Control, Ph.D. Dissertation, Department of Aeronautics and Astronautics, Massachusetts Institute of Technology, November 2004.

[29] BENSON, D. A., HUNTINGTON, G. T., THORVALDSEN, T. P., and RAO, A. V. "Direct Trajectory Optimization and Costate Estimation via an Orthogonal Collocation Method," presented as paper AIAA 2006-6358 at the AIAA Guidance, Navigation, and Control Conference, Keystone, Colorado, August 21-24, 2006.

[30] BENSON, D. A., HUNTINGTON, G.T., THORVALDSEN, T.P., and RAO, A. V. "Direct Trajectory Optimization and Costate Estimation via an Orthogonal Collocation Method," Journal of Guidance, Control, and Dynamics, Vol. 29, No. 6, November-December 2006, pp. 1435-1440.

[31] HUNTINGTON, G. T. and RAO, A. V. "Optimal Spacecraft Formation Configuration Using a Gauss Pseudospectral Method," Proceedings of the 2005 AAS/AISS Spaceflight Mechanics Meeting, AAS Paper 05-103, Copper Mountain, Colorado, January 23-27, 2005.

[32] HUNTINGTON, G. T. and RAO, A. V. "Optimal Reconfiguration of a Spacecraft Formation via a Gauss Pseudospectral Method," Proceedings of the 2005 AAS/AIAA Astrodynamics Specialist Conference, AAS Paper 05-338, Lake Tahoe, California, August 7-11, 2005.

[33] HUNTINGTON, G. T., BENSON, D. A., and RAO, A. V. "Post-Optimality Evaluation and Analysis of a Formation Flying Problem via a Gauss Pseudospectral Method," Proceedings of the 2005 AAS/AIAA Astrodynamics Specialist Conference, AAS Paper 05-339, Lake Tahoe, California, August 7-11, 2005.

[34] WERTZ, J. R. and LARSEN, W. J. Space Mission Analysis and Design, 3rd Edition, Microcosm Press, El Segundo, CA and Kluwer Academic Publishers, Dordrecht, The Netherlands, 1999, p. 694.

[35] BATTIN, R.H. An Introduction to the Mathematics and Methods of Astrodynamics, AIAA Press, New York, 1987, pp. 490-493 and p. 119.

[36] HARGRAVES, C. R. and PARIS, S. W. "Direct Trajectory Optimization Using Nonlinear Programming and Collocation," Journal of Guidance, Control, and Dynamics, Vol. 10, No. 4, 1987 , pp. 338-342.

[37] HUGHES, S. "Formation Tetrahedron Design for Phase 1 of the Magnetospheric Multiscale Mission," Flight Dynamics Analysis Branch, NASA Goddard Space Flight Center, 2004. 
[38] ROBERT, P., et.al. "Tetrahedron Geometric Factors," Analysis Methods for Multi-Spacecraft Data (G. Paschmann and P. Daly, eds.), Nordwijk, The Netherlands: ISSI Report SR-001, ESA Publications Division, 1998, pp. 323-348.

[39] HULL, D. G. Optimal Control Theory for Applications, Springer-Verlag, New York, 2003.

[40] KIRK, D. E. Optimal Control Theory: An Introduction, Dover Publications, 1998.

[41] PONTRYAGIN, L. S., BOLTYANSKII, V., GAMKRELIDZE, R., and MISCHENKO, E. The Mathematical Theory of Optimal Processes, New York: Interscience, 1962.

[42] DAVIS, P. Interpolation \& Approximation, Dover Publications, 1975.

[43] DAVIS, P. and RABINOWITZ, P. Methods of Numerical Integration, Academic Press, 1984.

[44] GILL, P.E. Users Guide for SNOPT Version 7, A Fortran Package for Large-Scale Nonlinear Programming, University of California, San Diego, La Jolla, CA, September 2004.

[45] CHOBOTOV, V. A., Ed. Orbital Mechanics, AIAA Press, New York, 1991, p. 18. 